\title{
THE ORION H II REGION AND THE ORION BAR IN THE MID-INFRARED
}

\author{
F. Salgado ${ }^{1}$, O. Berné ${ }^{2,3}$, J. D. Adams ${ }^{4}$, T. L. Herter ${ }^{4}$, L. D. Keller ${ }^{5}$, and A. G. G. M. Tielens ${ }^{1}$ \\ ${ }^{1}$ Leiden Observatory, University of Leiden, P.O. Box 9513, 2300 RA Leiden, Netherlands \\ ${ }^{2}$ Université de Toulouse; UPS-OMP; IRAP; Toulouse, France \\ ${ }^{3}$ CNRS; IRAP; 9 Av. colonel Roche, BP 44346, F-31028 Toulouse cedex 4, France \\ ${ }^{4}$ Astronomy Department, 202 Space Sciences Building, Cornell University, Ithaca, NY 14853-6801, USA \\ ${ }^{5}$ Department of Physics and Astronomy, Ithaca College, Ithaca, NY 14850, USA \\ Received 2015 October 23; revised 2016 July 20; accepted 2016 July 23; published 2016 October 17
}

\begin{abstract}
We present mid-infrared photometry of the Orion bar obtained with the Faint Object infraRed Camera for the SOFIA Telescope (FORCAST) on board SOFIA at 6.4, 6.6, 7.7, 19.7, 31.5, and 37.1 $\mu \mathrm{m}$. By complementing this observation with archival FORCAST and Herschel/PACS images, we are able to construct a complete infrared spectral energy distribution of the Huygens region in the Orion nebula. Comparing the infrared images with gas tracers, we find that PACS maps trace the molecular cloud, while the FORCAST data trace the photodissociation region (PDR) and the H II region. Analysis of the energetics of the region reveal that the PDR extends for $0.28 \mathrm{pc}$ along the line of sight and that the bar is inclined at an angle of $4^{\circ}$. The infrared and submillimeter images reveal that the Orion bar represents a swept-up shell with a thickness of $0.1 \mathrm{pc}$. The mass of the shell implies a shock velocity of $\simeq 3 \mathrm{~km} \mathrm{~s}^{-1}$ and an age of $\simeq 10^{5}$ years for the $\mathrm{H}$ II region. Our analysis shows that the UV and infrared dust opacities in the $\mathrm{H}$ II region and the PDR are a factor 5 to 10 lower than in the diffuse interstellar medium. In the ionized gas, Ly $\alpha$ photons are a major source of dust heating at distances larger than $\simeq 0.06 \mathrm{pc}$ from $\theta^{1}$ Ori $\mathrm{C}$. Dust temperatures can be explained if the size of the grains is between 0.1 and $1 \mu \mathrm{m}$. We derive the photoelectric heating efficiency of the atomic gas in the Orion bar. The results are in good qualitative agreement with models and the quantitative differences indicate a decreased polycyclic aromatic hydrocarbon abundance in this region.
\end{abstract}

Key words: dust, extinction - HII regions - infrared: ISM - ISM: individual objects (M42)

\section{INTRODUCTION}

Dust grains are an important constituent of the interstellar medium (ISM) of galaxies. From young stellar objects to active galactic nuclei, most of the objects in the universe show the presence of dust either in emission or absorption. Moreover, dust plays a key role in star and planet formation, molecule formation due to surface chemical reactions and heating of the gas due to the photoelectric effect. In addition, dust emission provides a convenient tracer of star formation in galaxies (e.g., Calzetti et al. 2007, 2010).

There is a consensus that dust grains are mainly composed of two chemically different type of species: silicates and carbonaceous materials (Zubko et al. 2004; Draine \& Li 2007). The former being the most abundant in mass for large grains, and the latter being the dominant species for smaller grains. At the lower end of the size distribution of grains, large molecules known as polycyclic aromatic hydrocarbons (PAH), formed by carbon rings, produce emission in characteristic infrared bands from 3 to $20 \mu \mathrm{m}$ (Tielens 2008). While the general properties of the dust grains in the diffuse ISM have been well studied, dust grain properties are expected to change with the environment. In particular, measurements of the extinction curve along different lines of sight in the Galaxy (Cardelli et al. 1989) reveal changes that are consistent with changes in the size distribution of dust grains. This finding is further supported by studies, such as that of Stepnik et al. (2003), which have also demonstrated variations in the dust size distribution and in the abundance measured as the gas-todust ratio. Recently, the Planck survey identified systematic variations in the submillimeter characteristics of interstellar dust, pointing toward the importance of dust evolution in the ISM (Planck Collaboration et al. 2014a). Changes in the size distribution of dust grains are expected from a theoretical perspective. Studies by Ormel et al. $(2009,2011)$ show that dust grains can coagulate in cold and dense molecular clouds, thus changing the size distribution. On the other hand, small dust grains and PAHs can be destroyed by the radiation field of stars. Furthermore, not only the size distribution but also the chemical composition of dust grains is expected to change with the environment (e.g., Henning 2010).

The extreme environments of regions of massive star formation may, in particular, be very conducive to dust processing. The study of dust around massive star-forming regions has been of major interest since the beginning of modern infrared and radio studies. In recent years, the study of dust in $\mathrm{H}$ II regions has increased; mostly driven by the increase of observations at infrared wavelengths due to Spitzer, Herschel, and most recently the Space Observatory for Infrared Astronomy (SOFIA). Deharveng et al. (2010), Anderson et al. (2012), Paladini et al. (2012), and Pellegrini et al. (2009) have studied the properties of dust in large evolved H II regions using combined data sets from Spitzer, Herschel, and APEX. Arab et al. (2012) and Salgado et al. (2012) have revealed the importance of dust variations in individual $\mathrm{H} \mathrm{II}$ regions. However, most of the data covered the far-infrared/ submillimeter, because - until recently-Spitzer/MIPS was the only instrument but with limited angular resolution $\left(6^{\prime \prime}\right)$ at midinfrared wavelengths. Now that SOFIA is in operation, moderate spatial resolution $\left(3^{\prime \prime}-3\right.$ !" 6 , depending on the wavelength) studies of dust emission from $\mathrm{H}$ II regions have come in reach. First, results have been published on dusty H II regions Salgado et al. (2012), Hirsch et al. (2012), and Lau et al. $(2013,2014)$. Here, we analyze data from the Faint Object infraRed Camera for the SOFIA Telescope (FORCAST) of the prototypical H II region, Orion (M42), from Shuping et al. 
(2012) more deeply to determine the characteristics of dust associated with the ionized gas and in the neutral photodissociation region (PDR).

The Orion Nebula (M42, NGC1976) is the closest (414 pc, Menten et al. 2007) and the most extensively studied region of massive star formation in the Galaxy. The overall structure of the Orion nebula has been well studied at visible wavelengths (O’Dell 2001; O’Dell et al. 2008, p. 544; O’Dell \& Harris 2010). In the center of the nebula, four young massive stars known as the Trapezium have photoionized the surrounding gas, creating an H II region; the global ionization structure is dominated by the most massive of the four stars, $\theta^{1}$ Ori C (spectral type $\sim 07-05 V$, Kraus et al. 2007; Stahl et al. 2008). The $\mathrm{H}$ II region is partially confined in a concave structure open toward the line of sight of the observer. A thin $(0.05 \mathrm{pc})$ and dense $\left(\sim 10^{5} \mathrm{~cm}^{-3}\right)$ PDR is located between the background molecular cloud and the ionized gas. Due to the difference in pressure between the PDR and the ionized gas, material flows toward $\theta^{1}$ Ori C (O'Dell \& Bally 1999; O'Dell 2001). At approximately $2^{\prime}$ southeast of the Trapezium the ionization front changes into an almost edge-on geometry producing the Orion bar feature, the prototypical PDR (Tielens et al. 1993; Hollenbach \& Tielens 1997). While several H II/ $\mathrm{PDR} /$ molecular clouds have been studied, Orion is still a cornerstone in understanding the interaction of newly formed massive stars with the surrounding gas and dust.

Previous ground-based studies of Orion in the mid-infrared regime have focused on the active star-forming core, including the $\mathrm{BN} / \mathrm{KL}$ object, and the region close to the Trapezium (Shuping et al. 2004; Robberto et al. 2005; Smith et al. 2005). These studies show a rich and complex structure, with diffuse extended emission, arcs, streaks, and emission associated with proplyds. In addition, ground-based and space-based studies on the spatial variations of the unidentified infrared bandsgenerally attributed to Polycyclic Aromatic Hydrocarbon molecules - have been reported by Cesarsky et al. (2000), Kassis et al. (2006), Galliano et al. (2008), and Haraguchi et al. (2012). Recently, Arab et al. (2012) studied the far-infrared emission of Orion using Herschel/PACS and SPIRE photometry. They compared Spitzer and Herschel data and found a shift in distance between the Orion bar as seen by Herschel and the IRAC $8 \mu \mathrm{m}$ emission. Here, we will combine this data with the mid-infrared observations of the Orion $\mathrm{H}$ II region obtained by the FORCAST instrument (Herter et al. 2012) on board of Stratospheric Observatory for Infrared Astronomy (SOFIA).

In Section 2, we provide a description of the new FORCAST observations, as well as a summary for optical, infrared, and submillimeter data from the literature used here. In Section 3, we give a description of the FORCAST images by comparing them with gas tracers. Together with Herschel/PACS farinfrared photometry, we construct spectral energy distributions (SEDs). The SEDs are further analyzed by using a two component modified blackbody model. In Section 4, the parameters derived from the SED fit are used to study the geometry of the Orion bar and the properties of the dust grains in the ionized gas and the photodissociation region (PDR). In Section 5, we provide an explanation for the observed temperature of the dust in the ionized gas, as well as for the dust properties observed in Orion. We compare our results to previous works from the literature putting our results in the broader context of $\mathrm{H}$ II region evolution. Finally, in Section 6, we summarize our work and provide conclusions.

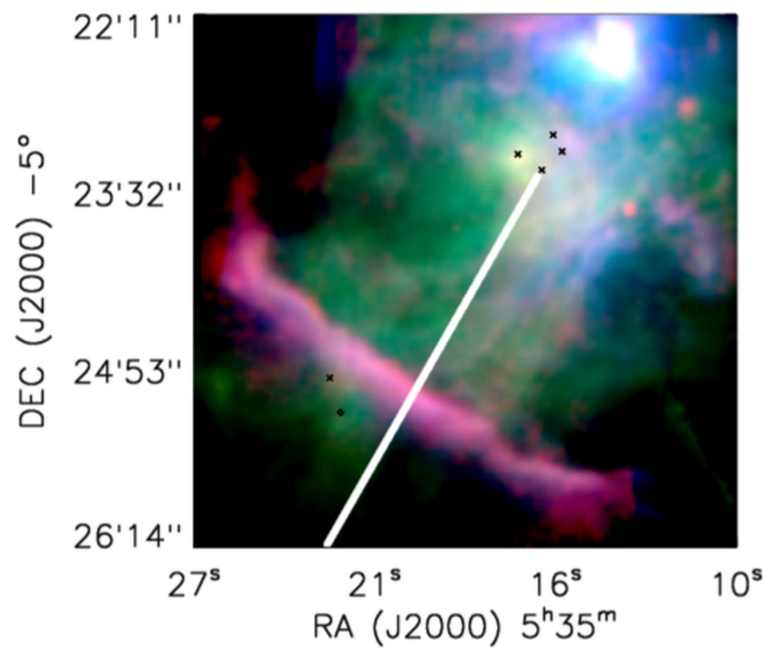

Figure 1. False color image for the FORCAST central region (blue 7.7, green 19.7, red 37.1). The Trapezium stars are marked with crosses. The Orion bar is prominent in the southeast while the $\mathrm{BN} / \mathrm{KL}$ region dominates the northwest (see text for details). The white line shows the cross-cut displayed in Figure 4.

\section{OBSERVATIONS AND DATA}

Two sets of observations were obtained by FORCAST on board SOFIA during the Short Science flight series: the first one is centered in the Trapezium stars and has been presented in Shuping et al. (2012), with a field of view (FOV) of roughly $3^{\prime} \times 3^{\prime}$ the images cover most of the $\mathrm{H}$ II region in the center of Huygens nebula (Figure 1). The second set of images are centered in the Orion bar with a similar FOV (Figure 1). The observations were made using FORCAST (Herter et al. 2012) on the $2.5 \mathrm{~m}$ telescope on board SOFIA. FORCAST is a $256 \times 256$ pixel dual-channel, wide-field mid-infrared camera sensitive from 5 to $40 \mu \mathrm{m}$ with a plate scale of $0 . / 768$ pixel $^{-1}$ and FOV of $3.4 \times 3 ! 2$. The two channels consist of a shortwavelength camera (SWC) operating from 5 to $25 \mu \mathrm{m}$ and a long wavelength camera (LWC) operating from 28 to $40 \mu \mathrm{m}$. An internal dichroic beam-splitter enables simultaneous observation from both long- and short-wavelength cameras. A series of bandpass filters are used to image at selected wavelengths.

The Orion bar data was taken over three flights: 2010 December 01, 2010 December 04, and 2010 December 08 with FORCAST. Dichroic mode was used to simultaneously observe in a number of filters: $19.7 / 31.4$, 19.7/37.1, 7.7/ 37.1, 6.6/37.1, and 11.3/37.1. The direct (single channel) mode was also employed for filters: 6.4, 6.6, 7.7, 11.3, and 37.1 microns. A southern region of the Orion bar was also observed in the dichroic mode with the 19.7/31.4 and 19.7/37.1 pairs. Data were reduced and calibrated as discussed in Herter et al. (2013).

A small rotation is seen in our Orion bar centered data for the 7.7 and the $31.7 \mu \mathrm{m}$ images, when comparing to the Trapezium centered images. Since no obvious point sources are detected in most of our images, another procedure must be performed to correct for this effect. At each wavelength, the images are rotated in discrete steps of 0.1 from $-4^{\circ}$ to $4^{\circ}$ and, by using a normalized cross-correlation estimator, a best expected rotation angle is obtained. The process was performed for all of our images, but only for the 7.7 and $31.7 \mu \mathrm{m}$ images is the rotation significant $\left(2^{\circ}\right)$. We checked the astrometry of our data after the correction is applied by comparing the $7.7 \mu \mathrm{m}$ to the $8 \mu \mathrm{m}$ 
Spitzer/IRAC image and the agreement is good to within about $1^{\prime \prime}$. Finally, the images were combined by averaging the flux in the region of overlap and scaling the full images accordingly.

We checked the relative flux calibration of the FORCAST images by comparing them with ISOCAM-LWS spectra (Cesarsky et al. 2000). The agreement is good in all bands. Using the ISOCAM-LWS spectra, we estimate the contribution from the [S III] $18.7 \mu \mathrm{m}$ line to the $19.7 \mu \mathrm{m}$ flux to be less than $10 \%$ at the ISOCAM-LWS positions.

Images from the PACS instrument (Poglitsch et al. 2010) on board of the Herschel Space Observatory (Pilbratt et al. 2010) at 70 and $160 \mu \mathrm{m}$ were obtained by Abergel et al. (2010) as a part of the "Evolution of interstellar dust" key program and have been presented in Arab et al. (2012). Centered at the Orion bar and with an FOV of about $14 \times 15^{\prime}$ the images overlap with our FORCAST images. Including the Herschel/ PACS images allows us to analyze the full dust SED at infrared wavelengths. The spatial resolution of the images is 5.'6 and 11 !" 3 at 70 and $160 \mu \mathrm{m}$, respectively.

Spectral cubes of infrared cooling lines [O I] at $63 \mu \mathrm{m}$ $145 \mu \mathrm{m}$ and [C II] at $157 \mu \mathrm{m}$ were observed by PACS and were published by Bernard-Salas et al. (2012). The spatial resolution of this data changes with the wavelength being $4 . .5$ at $63 \mu \mathrm{m}$ $10^{\prime \prime}$ at $145 \mu \mathrm{m}$ and $11^{\prime \prime}$ at $157 \mu \mathrm{m}$. The FOV is smaller than our FORCAST images overlapping only in the Orion bar.

Since the mid-infrared and far-infrared emission probes the dust emission, we complement our data set with low-resolution (10 A FWHM) optical spectroscopy from the Postdam MultiAperture Spectrograph (PMAS) in the PPAK mode at the $3.5 \mathrm{~m}$ telescope in Calar Alto Observatory (Sánchez et al. 2007). The data set consists of $\sim 8000$ spectra in the 3700-7000 $\AA$ range, centered in the Trapezium stars and covering $5^{\prime} \times 5^{\prime}$. Although the data was taken under non-photometric conditions, the spatial resolution is good enough for a spatial comparison with our infrared data. The astrometry was cross calibrated by comparing the data with Hubble Space Telescope images observed with WFPC.

We extracted the integrated flux and peak emission for some spectral lines at each pixel position, thus we created 2D maps for the most important lines that trace gas physical properties such as $\mathrm{H} \alpha, \mathrm{H} \beta$, [S II] $\lambda 6716,6731$, [O II] $\lambda 3727,3729$, [O III] $\lambda 4363$, [O III] $\lambda 4959,5007$, and the [O I] at $6300 \AA$. In our analysis, we studied the spatial distribution of [O I] as a tracer of the ionization front, and we neglect possible contamination due to sky emission. ${ }^{6}$

Rotational transition lines of ${ }^{13} \mathrm{CO}$ and $\mathrm{C}^{18} \mathrm{O}$ in the $J=3 \rightarrow 2$ transition were observed by the James Clerck Maxwell Telescope (JCMT) using HAARP/ACSIS, published by Buckle et al. (2012). The resolution is close to $15^{\prime \prime}$ and the FOV overlaps with our data set.

Simón-Díaz et al. (2006) studied the spectra of eight stars including $\theta^{1}$ Ori C. They determined a $T_{\text {eff }}=39,000 \pm 1000 \mathrm{~K}$ and a $\log g=4.1$ corresponding to an $05.5 \mathrm{~V}-\mathrm{O} 6 \mathrm{~V}$ star in the scale of Martins et al. (2005). By interpolating the values of Table 1 in Martins et al. (2005), we derived a stellar luminosity of $L=2.23 \times 10^{5} L_{\odot}$ and the number of ionizing photons $N_{\text {ion }}=1.06 \times 10^{49} \mathrm{~s}^{-1}$. The second most massive star is $\theta^{1}$ Ori D a B0.5V star Simón-Díaz et al. (2006), but with an

\footnotetext{
6 The [O I] $\lambda 6300 \AA$ flux is expected to be only $20 \%$ larger than that of the sky and, due to the low spectral resolution of the PMAS instrument, both lines are not easily separable. However, the contribution from the SKY emission, for the purpose of the analysis presented here, can be treated as random noise (or rather a baseline contribution to the emission). The emission at the position of interest for our analysis are well above the $3 \sigma$ confidence level.
}

effective temperature more similar to an $\mathrm{O} 9$ star. The star $\theta^{1}$ Ori $\mathrm{A}$ is a B0.5 star. Using the values for the stars, we integrated the spectra of the stellar models (Martins et al. 2005 and Kurucz 1993 for B0.5V stars) from 912 to $1000 \AA$ to obtain a total FUV luminosity in the range of $1.4-2.7 \times 10^{5} L_{\odot}$. The properties of the Trapezium stars are summarized in Table 2.

\section{ANALYSIS}

\subsection{An Overall View of the Orion Nebula}

The Orion Nebula is one of the most studied massive starforming regions. At optical wavelengths, the gas in the Orion Nebula shows a complex structure (e.g., Doi et al. 2004). In addition to stars, the diffuse emission, the ionization front, as well as the proplyds and jets have been thoroughly studied. As can be seen in Figures 1 and 2, the FORCAST midinfrared images show an intricate morphology that changes with wavelength. In particular, the $6.4,6.6$, and $7.7 \mu \mathrm{m}$ images (PAH images) show the Orion bar, the Trapezium stars, and the Ney-Allen nebula. In the $19 \mu \mathrm{m}$ band, in addition, the Herbig-Haro object $\mathrm{HH} 203$, is clearly visible and there is also emission associated with the stars, $\theta^{2}$ Ori A and $\theta^{2}$ Ori B. The BN/KL object (north of the Trapezium), the brightest region in the FORCAST images, is saturated in the Herschel/PACS bands.

Close to the Trapezium stars and toward the southwest there is bright extended emission in all PAH and mid-infrared images, this emission seems co-spatial with the peak in emission in radio and $\mathrm{H} \alpha$ images (Dicker et al. 2009). Southeast of the Trapezium, extended emission and substructure is seen as "filaments," arcs, and "ripples" toward the Orion bar in all the FORCAST images. We note that this spatial structure is not correlated with the optical extinction maps derived from $\mathrm{H} \alpha / \mathrm{H} \beta$ maps (O'Dell \& Yusef-Zadeh 2000; Sánchez et al. 2007), which is consistent with the idea that most of the extinction toward the region is produced by a foreground cloud. Instead, these structures are thought to be perturbations in the surface of the background PDR (Shuping et al. 2012).

The high resolution and large FOV of the FORCAST images together with the large amount of observations from the literature allow us to directly compare the spatial distribution of dust emission with different gas tracers. However, a close comparison between the IR and optical maps is hampered by the presence of emission associated with the PDR and HH203/204 jets penetrating into the ionized volume. Comparison of the 19.7 and $7.7 \mu \mathrm{m}$ maps reveals a similarity for some spatial structures, which we interpret as being associated with the PDR. Nevertheless, several regions bright in $19.7 \mu \mathrm{m}$ emission do not show up in the $7.7 \mu \mathrm{m}$ image. Hence, we consider that this part of the $19.7 \mu \mathrm{m}$ emission is associated with dust in the ionized gas rather than in the background PDR. To bring this out, we have subtracted the $7.7 \mu \mathrm{m}$ map from the $19.7 \mu \mathrm{m}$ map scaled to the intensity ratio in the Orion bar PDR, the result is shown in Figure 3. Perusing the velocity dependent $\mathrm{H} \alpha$ maps (Doi et al. 2004), we recognize a resemblance to several structural features in the subtracted $19.7 \mu \mathrm{m}$ emission with the $\mathrm{H} \alpha$ emission in the range of $0-8 \mathrm{~km} \mathrm{~s}^{-1}$ (with respect to LSR, Doi et al. 2004) particularly directly north of the bar. However, not surprisingly, there is no one-to-one correspondence as gas and dust emission processes differ in their density and temperature 


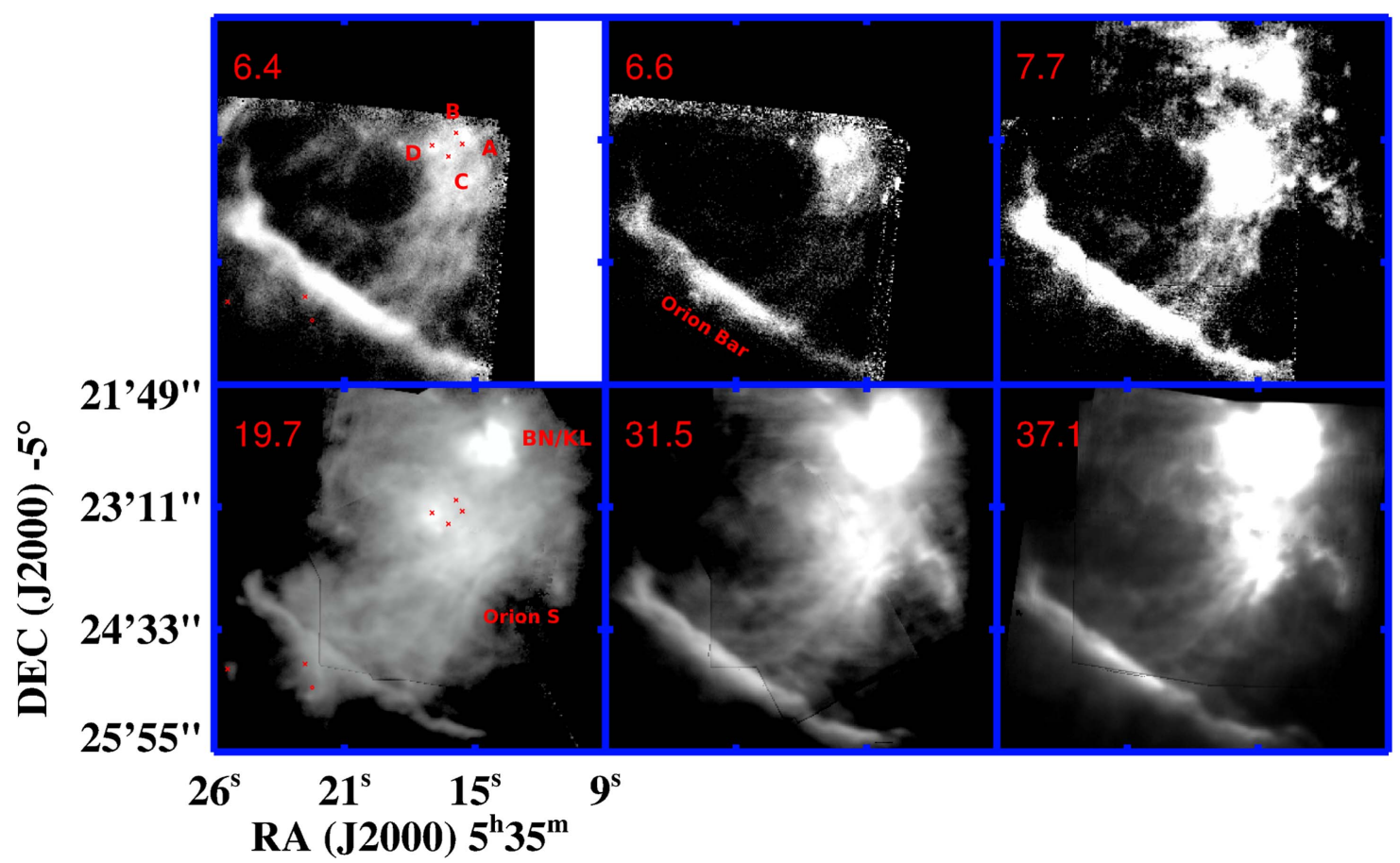

Figure 2. Complete data set of FORCAST images at 6.4, 6.6, 7.7, 19.7, 31.5, and $37.1 \mu \mathrm{m}$. Red crosses correspond to the Trapezium stars and $\theta^{2}$ Ori A and $\theta^{2}$ Ori B. The diamond marks the location of HH 203/204.

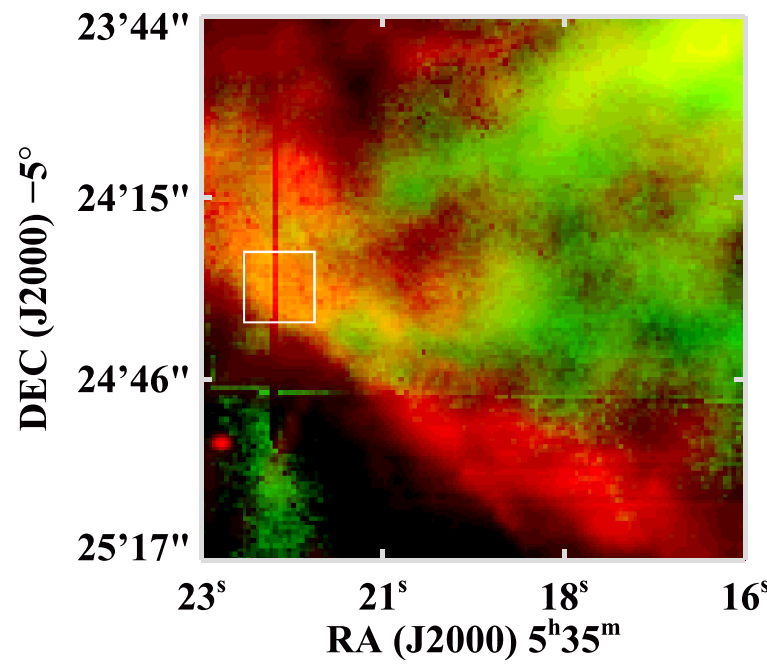

Figure 3. Comparison between the $19 \mu \mathrm{m}$ PDR-subtracted emission (green) and $\mathrm{H} \alpha$ at $8 \mathrm{~km} \mathrm{~s}^{-1}$ from (Doi et al. 2004) (red). While there is no one-to-one correspondence between the two images, some features are co-spacial. The white box marks one of the regions where there is correspondence between dust and gas emission.

dependence. Moreover, we also recognize that decoupling of gas and dust seems to be a common phenomena in blister $\mathrm{H}$ II regions (Ochsendorf et al. 2014b) and a one-to-one correspondence is not expected. We also notice that some of the dust emission can be related to the "Big Arc," in the terms of Doi et al. (2004). Furthermore, the $19.7 \mu \mathrm{m}$ emission is not related to the molecular cloud (see the 70 and $160 \mu \mathrm{m}$, Figure 4). While some of the emission can be related to the PDR (as traced by PAH emission, Figure 4), the rest of the emission seem to be associated with ionized gas (see Figure 2 and, for instance, O’Dell \& Doi 2003).
At longer wavelengths, the PACS images show the Orion bar as a bright feature, but the brightest emission is associated with the Orion molecular cloud (OMC-1). Note that, unlike the PAH and mid-infrared emission, the region close to the Trapezium stars does not show strong, associated, far-infrared emission in the PACS bands. By comparing the far-infrared with the optical images, the only similarities are found toward the ionization fronts in the direction of the Orion bar and Orion S.

To further analyze the spatial distribution of the emission, we extracted cross-cuts starting from $\theta^{1}$ Ori $C$ to deep behind the Orion bar at the position shown in Figure 1. The extracted profiles are shown in Figure 4. A general trend is that the dust emission is bright close to the Trapezium and drops with distances up to $0.19 \mathrm{pc}$ where the Orion bar is prominent (see Section 3.2). A closer look reveals the presence of small structures in all the FORCAST bands at $10^{\prime \prime}, 30^{\prime \prime}$, and $80^{\prime \prime}$, these structures are more prominent at $19.7 \mu \mathrm{m}$.

\subsection{The Orion Bar}

All images show a prominent structure to the southwest associated with the well-known ionization front, the Orion bar (Figures 1 and 2). However, there are subtle variations in the maps. Specifically, the position of the peak emission changes with wavelength (Table 3). Indeed, comparison of the $\mathrm{H} \alpha$, [O I] $6300 \AA$, PAHs, $\mathrm{H}_{2}$, and ${ }^{13} \mathrm{CO}$ emission reveal the layered structure expected for an edge-on PDR (Figure 4; Tielens et al. 1993). The dust emission show a similar stratified structure, with shorter wavelengths peaking closer to the Trapezium stars as compared with longer wavelength images, consistent with heating by the Trapezium stars of an edge-on slab (Werner et al. 1976; Tielens et al. 1993; Arab et al. 2012). The ionization front, as traced by the [O I] 6300 A profile, show a sharp rise and a "winglike" decay, but the peak is located closer to $\theta^{1}$ Ori $\mathrm{C}$ at $113^{\prime \prime}$ $(0.23 \mathrm{pc})$. The $\mathrm{H} \alpha$ peak emission is located even closer to 

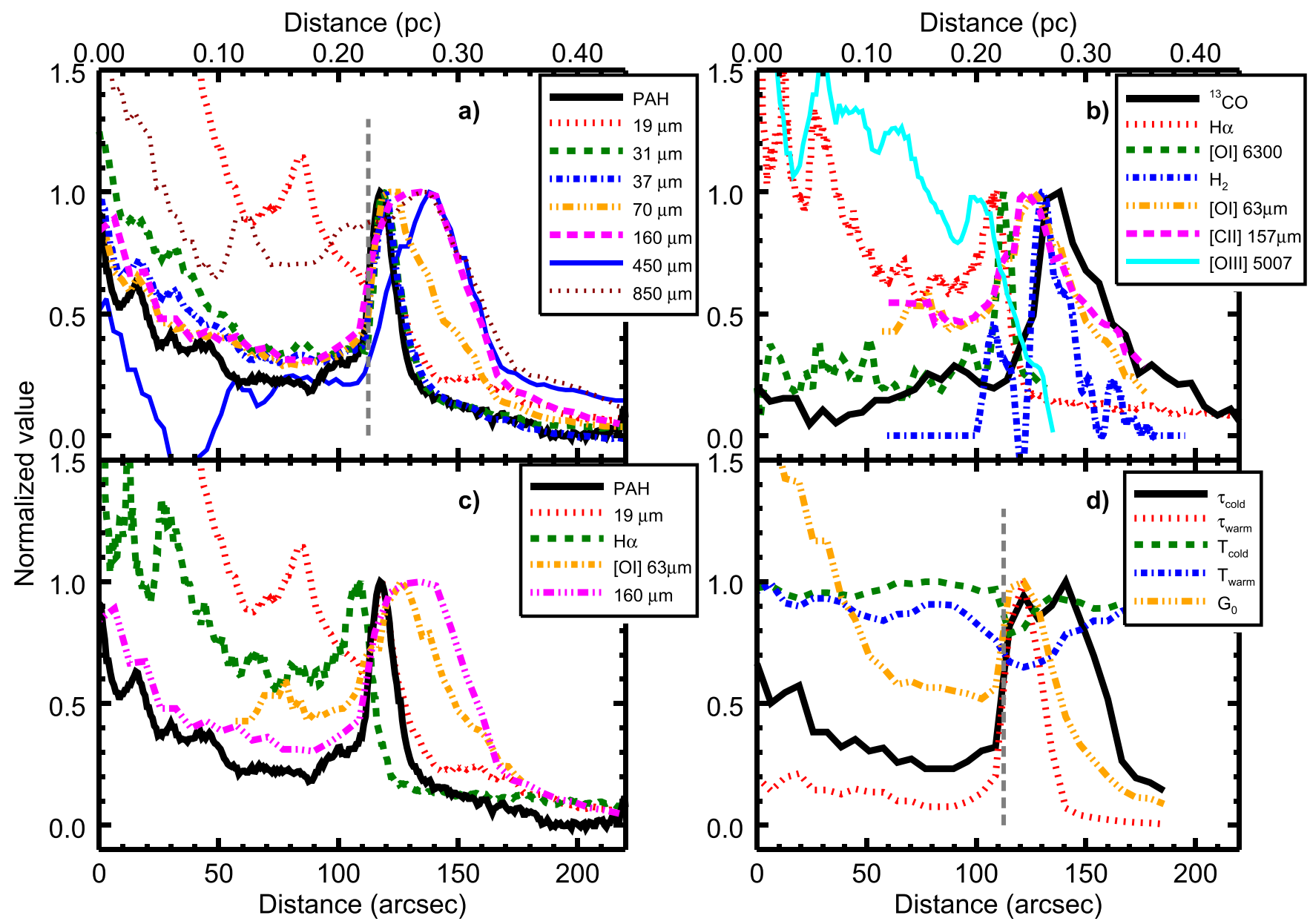

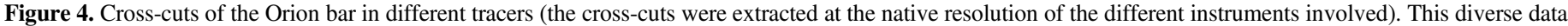

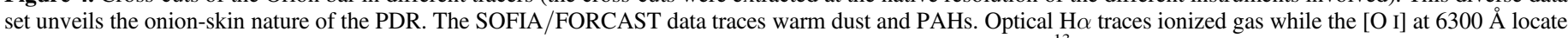

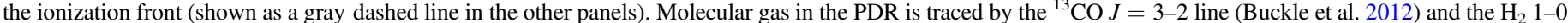

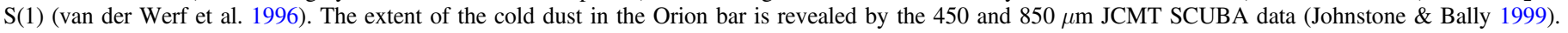

$\theta^{1}$ Ori $\mathrm{C}$ at $109^{\prime \prime}(0.22 \mathrm{pc})$. The PAH, 31 and $37 \mu \mathrm{m}$ images show profiles with a sharp rise and a peak at $118^{\prime \prime}(0.24 \mathrm{pc})$. The $19.7 \mu \mathrm{m}$ image shows, in addition, a separate peak associated with the $\mathrm{H}$ II region but there is no clear counterpart in any other ionized optical line. At longer wavelengths, the $70 \mu \mathrm{m}$ also shows a sharp rise at the bar position but the peak is broader than at midinfrared wavelengths. This broadening trend seems to continue in the $160 \mu \mathrm{m}$ data, but this trend is clearly an effect of the lower resolution of PACS at $160 \mu \mathrm{m}$. The ${ }^{13} \mathrm{CO}$ show a broader "Gaussian-like" profile with peaks at 138 " $(0.28 \mathrm{pc})$, respectively. At $450 \mu \mathrm{m}$, the peak of the emission has shifted inward and occurs at the ${ }^{13} \mathrm{CO}$ peak. ${ }^{7}$ The ground-based $850 \mu \mathrm{m}$ data, on the other hand, shows an emission peak that encompasses both the mid-infrared and the $450 \mu \mathrm{m}$ peak (Figure 4). The "shoulder" seen in the $850 \mu \mathrm{m}$ images at distances less than $0.2 \mathrm{pc}$ is, most likely, free-free emission produced in the ionized gas.

Assuming a constant PDR density of $10^{5} \mathrm{~cm}^{-3}$ (Simon et al. 1997), we can quantify the location of the different emission peaks in terms of the total column density. We locate the surface of the PDR with the emission peak of the PAH features, at 117 ".5 from $\theta^{1}$ Ori $\mathrm{C}$ (see Table 3 ). The mid-infrared dust continuum peaks slightly deeper into the PDR. The $160 \mu \mathrm{m}$

\footnotetext{
Some negative values were found in the $450 \mu \mathrm{m}$ image so we added a constant to the data.
}

emission shows a rather broad peak, running from $120^{\prime \prime}$ to $140^{\prime \prime}$, while the submillimeter emission peaks at about $140^{\prime \prime}$. For comparison, the ${ }^{13} \mathrm{CO}$ peak is located at a column density of $N_{\mathrm{H}}=12.7 \times 10^{21} \mathrm{~cm}^{-2}$, while the peak in $\mathrm{H}_{2}$ emission is located at $20^{\prime \prime}$ from the ionization front, roughly $0.04 \mathrm{pc}$ or at a depth of $N_{\mathrm{H}}=7.74 \times 10^{21} \mathrm{~cm}^{-2}$. At a distance of $200^{\prime \prime}$ $(0.4 \mathrm{pc})$, the emission in all the bands has dropped to less than $20 \%$ of the peak emission at the bar. The submillimeter data conclusively show that the Orion bar is limited in extent in the molecular cloud to $\simeq 140^{\prime \prime}$ or a total column of $N_{\mathrm{H}}=13$. $9 \times 10^{22} \mathrm{~cm}^{-2}$. The Orion bar is a shell bounded on one side by the ionization front and on the other side (likely) by the shock front running into the molecular cloud.

In neutral regions, the cooling is dominated by three forbidden transitions lines: [O I] at $63 \mu \mathrm{m}$ [C II] at $157 \mu \mathrm{m}$ and [O I] at $145 \mu \mathrm{m}$. The [O I] $63 \mu \mathrm{m}$ and the [C II] $157 \mu \mathrm{m}$ line peaks are located at a distance of $125^{\prime \prime}(0.25 \mathrm{pc})$ corresponding to a column density of $N_{\mathrm{H}}=4.65 \times 10^{21} \mathrm{~cm}^{-2}$. This is slightly deeper in the Orion bar than the PAH and mid-infrared emission peaks (Figure 4). We also note that clumps seen in the [O I] and [C II] IR lines by Bernard-Salas et al. (2012) are also evident in mid-infrared continuum emission. The [O I] and [C II] structures are, however, located somewhat deeper than clumps in the dust and PAH emission maps (Figure 5). 


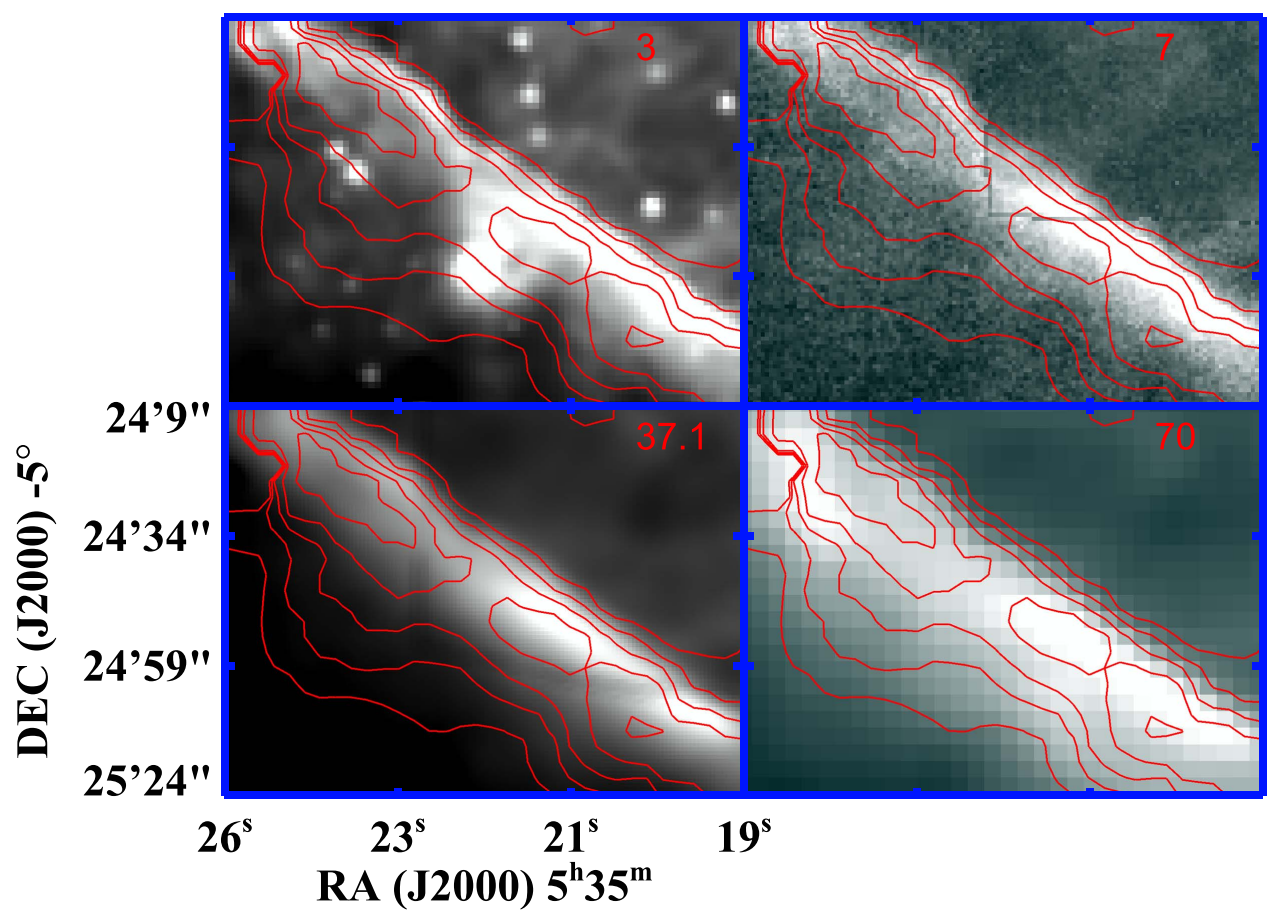

Figure 5. Upper left: Spitzer/IRAC $3 \mu \mathrm{m}$ image at the Orion bar. Upper right: FORCAST $7.7 \mu \mathrm{m}$ image at the Orion bar. Lower left: FORCAST $37.1 \mu \mathrm{m}$ image at the Orion bar. Lower right: PACS $70 \mu \mathrm{m}$ image at the Orion bar. The red contours are the [O I] $63 \mu \mathrm{m}$ integrated emission, the peak of the line emission is located deeper into the PDR as compared to the dust tracers.

Table 1

FORCAST and PACS Angular Resolution

\begin{tabular}{lc}
\hline \hline Wavelength $(\mu \mathrm{m})$ & FWHM $\left(^{\prime \prime}\right)$ \\
\hline 6.4 & 2.9 \\
6.6 & 3.0 \\
7.7 & 2.7 \\
19.7 & 2.9 \\
31.5 & 3.4 \\
37.1 & 3.6 \\
70 & 5.6 \\
160 & 11.3 \\
\hline
\end{tabular}

Table 2

Properties of the Central Stars (Martins et al. 2005)

\begin{tabular}{lcccc}
\hline \hline Star & $\begin{array}{c}\text { Spectral } \\
\text { Type }\end{array}$ & $\begin{array}{c}\text { Ionizing } \\
\text { Photon Flux }\end{array}$ & $\begin{array}{c}\text { Luminosity log } \\
\left(L / L_{\odot}\right)\end{array}$ & $\begin{array}{c}\text { FUV Luminos- } \\
\text { ity } \log \left(L / L_{\odot}\right)\end{array}$ \\
\hline$\theta^{1}$ Ori A & B0.5V & 47.36 & 4.52 & 4.61 \\
$\theta^{1}$ Ori B & B1V & 47.06 & 4.42 & $\ldots$ \\
$\theta^{1}$ Ori C & O4V- & 48.96 & $5.68-5.31$ & $5.26(\mathrm{O} 5.5)$ \\
& O6V & & & 4.61 \\
$\theta^{1}$ Ori D & B0.5V & 47.36 & 4.52 & \multirow{2}{*}{4} \\
\hline
\end{tabular}

\subsection{SED Fitting}

The SOFIA/FORCAST data provide, for the first time, full spectral coverage of the SED of the dust emission in the Orion bar with high angular resolution. In fact, the FORCAST filters are well matched to the peak of the SED, which shifts from about 20 to $70 \mu \mathrm{m}$ over this region. In order to analyze the SED of our images, the FORCAST and the PACS $70 \mu \mathrm{m}$ images were convolved to the PACS $160 \mu \mathrm{m}$ resolution assuming a Gaussian PSF and using the values in Table 1 for the FWHM. The images were regridded to a common pixel scale of $6 ! " 4$, the
Table 3

Spatial Emission Peaks

\begin{tabular}{|c|c|c|c|}
\hline $\begin{array}{l}\text { Wavelength } \\
(\mu \mathrm{m}) / \text { Line }\end{array}$ & Distance $\left({ }^{\prime \prime}\right)^{\mathrm{a}}$ & $\begin{array}{c}N_{\mathrm{H}}^{\mathrm{b}} \\
\left(\times 10^{21} \mathrm{~cm}^{-2}\right)\end{array}$ & comment \\
\hline 6.4 & $117.5 \pm 0.8^{\mathrm{c}}$ & 0 & PDR surface \\
\hline 6.6 & $117.5 \pm 0.8$ & 0 & $\ldots$ \\
\hline 7.7 & $117.5 \pm 0.8$ & 0 & $\ldots$ \\
\hline 19.7 & $118 \pm 0.8$ & 0.31 & $\ldots$ \\
\hline 31.5 & $119.5 \pm 0.8$ & 1.24 & $\ldots$ \\
\hline 37.1 & $120 \pm 0.8$ & 1.55 & $\ldots$ \\
\hline 70 & $125 \pm 3$ & 4.65 & $\ldots$ \\
\hline 160 & $135 \pm 6$ & 10.8 & $\ldots$ \\
\hline 450 & $138 \pm 6$ & 12.7 & $\ldots$ \\
\hline 850 & $138 \pm 6$ & 12.7 & $\cdots$ \\
\hline$[\mathrm{O} \mathrm{I}]_{63}$ & $125 \pm 10$ & 4.65 & $\ldots$ \\
\hline$[\mathrm{C} \mathrm{II}]_{157}$ & $125 \pm 10$ & 4.65 & $\ldots$ \\
\hline$[\mathrm{O} \mathrm{I}]_{6300}$ & $112.5 \pm 3$ & $\ldots$ & Ionization front \\
\hline $\mathrm{H}_{\alpha}{ }^{\mathrm{d}}$ & $109 \pm 0.2$ & $\ldots$ & Ionization bar \\
\hline $\mathrm{H}_{2}{ }^{\mathrm{e}}$ & 130 & 7.74 & $\begin{array}{c}\text { Peak of the } \\
1-0 \mathrm{~S}(1)\end{array}$ \\
\hline${ }^{13} \mathrm{CO}(3-2){ }^{\mathrm{f}}$ & $138 \pm 6$ & 12.7 & $\begin{array}{c}\text { Peak of low- } \\
J^{13} \mathrm{CO}\end{array}$ \\
\hline
\end{tabular}

Notes.

${ }^{\mathrm{a}}$ Measured from $\theta^{1}$ Ori C.

${ }^{\mathrm{b}} N_{\mathrm{H}}$ measured from the PDR surface, using the spatial extent and adopting a density of $1 \times 10^{5} \mathrm{~cm}^{-3}$ (Simon et al. 1997).

${ }^{c}$ Errors are the pixel scale of the images.

d Taken from HST images O'Dell \& Yusef-Zadeh (2000).

e Maps from van der Werf et al. (1996).

f Taken from Buckle et al. (2012).

native resolution of PACS at $160 \mu \mathrm{m}$. Figure 6 shows SEDs at a few selected positions. The SED peak changes with position from around $30 \mu \mathrm{m}$ close to the Trapezium and moving to $80 \mu \mathrm{m}$ behind the Orion bar. 


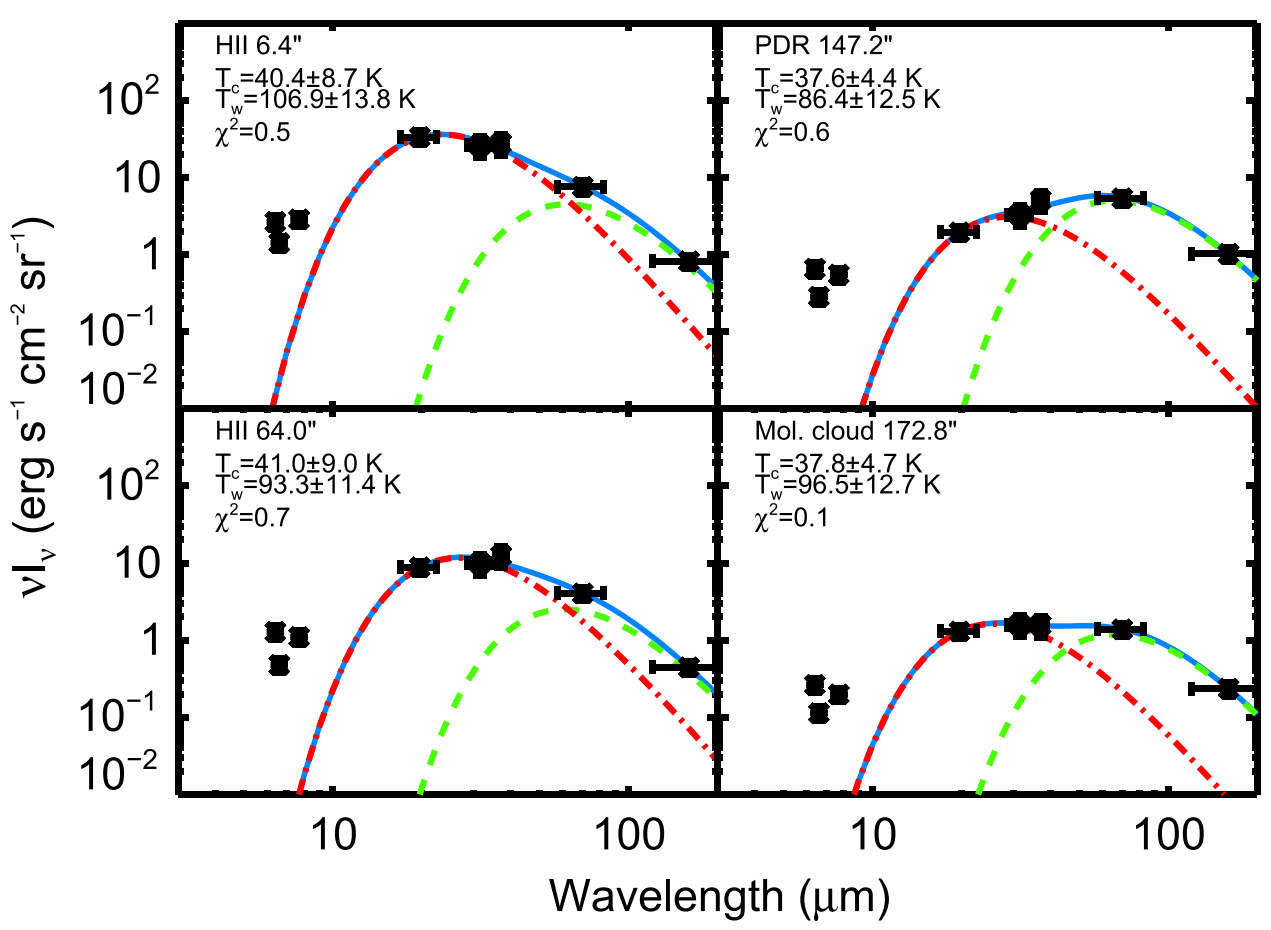

Figure 6. Examples of the SEDs at four positions. The results of the modified blackbody fits are also plotted.

The emission seen at wavelengths shorter than $\sim 10 \mu \mathrm{m}$ is expected to be produced by PAHs and very small grains in the background PDR and in the molecular cloud. Furthermore, The ISOCAM-CVF spectra (Cesarsky et al. 2000) clearly shows the PAH bands at 6.4 and $7.7 \mu \mathrm{m}$. As PAHs and very small grains are stochastically heated, we decided to exclude the emission at these wavelengths from the SED analysis. At wavelengths larger than $\sim 10 \mu \mathrm{m}$, the SEDs are very broad and show evidence of two separate components; particularly beyond the Orion bar. Indeed, modified blackbody fits to the SEDs at each pixel position with a spectral index of $\beta=1.8$ are not satisfactory. Modified blackbody fits with variable $\beta$ (between 0 and 3) yield better results in terms of the reduced $\chi^{2}$. However, the $\beta$ values are too low $(\beta<0.5)$, far from standard values.

The IR emission observed in the maps result from the contribution of grains located within the H II region, in the PDR and in the molecular cloud. When comparing the PACS $160 \mu \mathrm{m}$ emission with the ${ }^{13} \mathrm{CO}$ data, it is clear that there is a component associated with the Orion molecular cloud (see Figure 7). The mid-infrared emission observed by SOFIA/FORCAST and farinfrared emission from Herschel/PACS $70 \mu \mathrm{m}$ are tracing different components of the dust. This can clearly be seen at positions close to the Orion bar where the combination of two modified blackbody functions are needed to obtain a good description of the data (see Figure 6 for examples of the SEDs and fits). Therefore, we fitted all the data with two modified blackbody components assuming optically thin emission using $I_{\lambda}=\tau_{\text {warm }} \times B_{\lambda}\left(T_{\text {warm }}\right) \times\left(\lambda / \lambda_{0}\right)^{-\beta}+\tau_{\text {cold }} \times B_{\lambda}\left(T_{\text {cold }}\right)$ $\left(\lambda / \lambda_{0}\right)^{-\beta}$, where warm corresponds to $T_{\text {warm }}>70 \mathrm{~K}$ and cold to where $T_{\text {cold }}<70 \mathrm{~K}$. In order to limit the number of free parameters, we have fixed the exponent of the modified blackbody for both components $(\beta=1.8$, Planck Collaboration et al. 2011). The constants, $\tau_{\text {warm }}$ and $\tau_{\text {cold }}$, are the optical depth at a given reference wavelength (we adopt $\lambda_{0}=100 \mu \mathrm{m}$ ).
Dust grains can be either in equilibrium with the radiation field or stochastically heated by photons depending on the size of the grain and the strength of the radiation field. In Orion, due to the strength of the radiation field $\left(\sim 1 \times 10^{5} G_{0}\right.$, Section 4.1), the emission of dust particles is produced by dust particles in radiative equilibrium with the radiation field, irrespective of their size (except for PAHs). Indeed, small (carbonaceous) dust particles of $\gtrsim 10 \AA$ in size ( $\gtrsim 120 \mathrm{C}$ atoms) are expected to be in equilibrium with the radiation field (Draine \& Li 2001, 2007). The steep increase in density (and extinction) at the Orion bar produces a clear broadening of the SEDs. While large grains remain in equilibrium with the (lower) radiation field, small grains are stochastically heated. Therefore, deeper in the Orion bar PDR, the warm component is produced by small grains stochastically heated.

Figure 8 shows the temperature and optical depth maps for both components at the positions where our fit is reasonably good (reduced $\chi^{2}<3$ ). Clearly, PACS $160 \mu \mathrm{m}$ trace a cold dust component, while the FORCAST mid-infrared images trace a warm dust component. The contribution to the PACS $70 \mu \mathrm{m}$ emission is a combination of both the warm and the cold component (see also Figure 9). The cold dust component reveals a temperature in the $38-44 \mathrm{~K}$ range to the east and southeast of the Trapezium. At the Orion bar, the temperature decreases to values of about $33 \mathrm{~K}$. Toward Orion $\mathrm{S}$ and Orion $\mathrm{BN} / \mathrm{KL}$, the temperature is also low. Unfortunately, toward Orion $\mathrm{BN} / \mathrm{KL}$, the PACS images are saturated and the fit results are not reliable. In terms of the optical depth, the warm component shows a spatial correlation with the ionized gas emission and is fairly uniform (Figure 8(c)). An increase in $\tau_{\text {warm }}$ is seen toward Orion $\mathrm{S}$, the Orion bar and the BN/KL object. Variations in temperature for the warm component are large, ranging from $70 \mathrm{~K}$ at the Orion bar to values larger than $100 \mathrm{~K}$ in the ionized gas. Close to $\theta^{1}$ Ori $\mathrm{D}$, the temperature of the Ney-Allen nebula reaches values of $120 \mathrm{~K}$. A high temperature $(110 \mathrm{~K})$, as compared to its surrounding area, is 


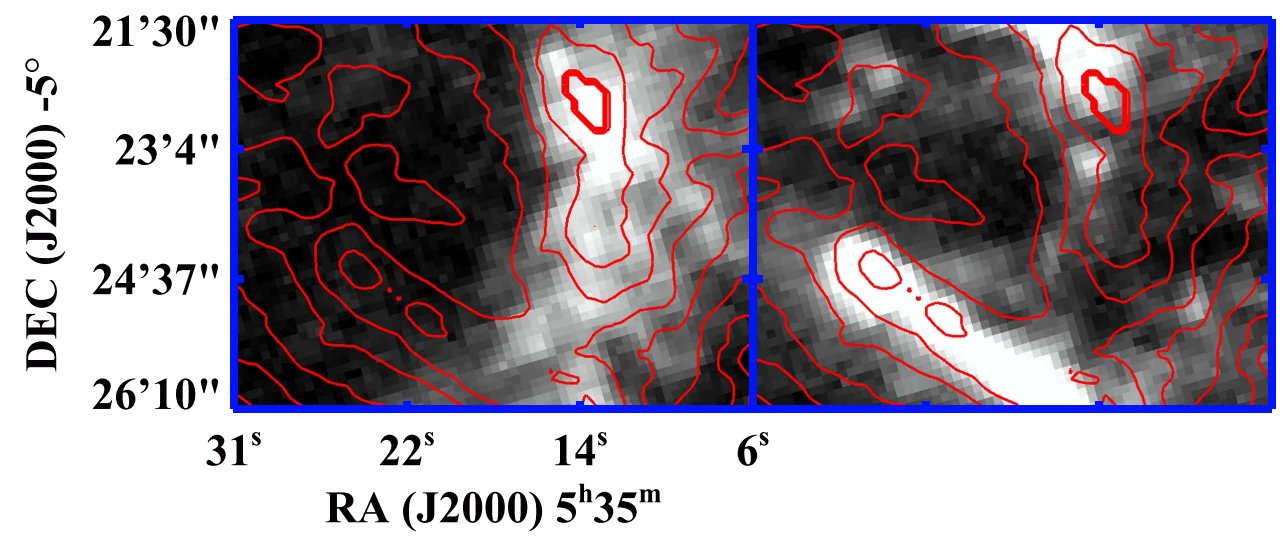

Figure 7. ${ }^{13} \mathrm{CO}$ data at two velocity components corresponding to $\mathrm{OMC}$ (left) and the Orion bar (right); the red contours are the PACS $160 \mu \mathrm{m}$ emission.

seen toward the Herbig-Haro object HH 203. The peak in $\tau_{\text {warm }}$ is located at the same distance as the $\mathrm{PAH} /$ mid-infrared peak $\left(120^{\prime \prime}\right)$, while the cold dust component show two peaks at $120^{\prime \prime}$ and $140^{\prime \prime}$.

Perusal of the optical depth maps reveals that the cold component is strongly concentrated toward the Orion Molecular Cloud Core OMC-1, and besides the BN/KL region, we also recognize Orion $\mathrm{S}$, but the Orion bar does not figure prominently. In contrast, the Orion bar is very evident in the optical depth map of the warm component. The temperature map provides a complementary view: the cold component reaches a particularly low temperature in the cold molecular cloud core-as evident in the temperature map of the cold component-as expected from the high column density associated with this feature. We also note that the highest temperatures for the cold component are present in the ionized gas toward the west of the Trapezium and also within a small pocket near $\theta^{2}$ Ori A. This same area around $\theta^{2}$ Ori $\mathrm{A}$ and HH 203 sticks out as particularly warm in the warm component map. At first sight, surprisingly, the Orion bar shows up in the temperature map of the warm component as a relatively cold feature compared to the much warmer material associated with the ionized gas. This reinforces the discussion in Section 3.1 that much of the mid-IR emission toward the north of the Orion bar is associated with dust in the ionized gas. We also note the increased presence of the warm dust component beyond the extent of the Orion bar traced in the submillimeter, which we attribute to dust associated with a thin layer of ionized gas that is quite evident in the optical and IR spectroscopic data (Rubin et al. 2011; Boersma et al. 2012).

The total dust emission was estimated by integrating the flux from the modified-blackbody fits. We can also assess the contribution from the cold and warm component to the overall IR emission. In the ionized zone, toward the Trapezium stars, the total luminosity is dominated by the warm component, being a factor of seven larger than the cold component on average. In the $\mathrm{H}$ II region, the ratio decreases with distance up to $115^{\prime \prime}$ - the Orion bar-where it suddenly increases. Beyond the surface of the Orion bar, the ratio decreases again, reaching a minimum of 0.6 at $\sim 150^{\prime \prime}$. The further increase beyond this minimum (toward the southeast) is not associated with the bar as outlined by the submillimeter emission.

As mentioned in Section 3.1, the infrared emission is dominated by the $\mathrm{BN} / \mathrm{KL}$ object, a region around the Trapezium and the Orion bar PDR. We focus on the Trapezium region and the bar, as the $\mathrm{BN} / \mathrm{KL}$ object is not covered in all of our images. We extracted the total luminosity in the region shown in Figure 9: one in a rectangular aperture of $158 \times 20^{\prime \prime}$ $\left(0.32 \times 0.04 \mathrm{pc}^{2}\right)$ at the Orion bar. The total luminosity at the surface of the Orion bar is $9.5 \times 10^{3} L_{\odot}$.

\section{RESULTS}

\subsection{Geometry of the Orion Bar}

The observed total infrared emission is a measure for the amount of stellar energy intercepted by the Orion bar PDR and we can use this value to determine the geometric dimensions of the bar. We will use the emission traced by the warm dust component to estimate the energy absorbed by the dust in the Orion bar region $\left(L_{\mathrm{IR}}=9.5 \times 10^{3} L_{\odot}\right)$. We can derive a value for the absorbing area at the surface of the PDR, i.e., $A_{\mathrm{abs}}=L_{\mathrm{IR}} /\left(L_{\star} / 4 \pi d^{2}\right)$. The minimum stellar luminosity of $1.4 \times 10^{5} L_{\odot}$ corresponds to the often adopted $G_{0}$ of $5 \times 10^{4}$ (Tielens \& Hollenbach 1985), while the twice higher luminosity of the Trapezium cluster (Section 2) results in $G_{0}$ that is double this value. In contrast, Marconi et al. (1998) derived a $G_{0}$ of $2.6 \times 10^{4}$ from the fluorescent, near-IR OI lines, originating in the ionization front. Adopting this latter value, the absorbing area is $0.09 \pm 0.02 \mathrm{pc}^{2}$. Adopting a rectangular geometry and using the measured size of the Orion bar $(0.32 \mathrm{pc})$ as the other side of the rectangle, we obtain a transverse size of $l_{\text {LOS }}=0.28 \pm 0.06 \mathrm{pc}$.

The line-of-sight extent can also be derived from observed atomic or molecular column densities in the Orion bar PDR, adopting a gas density. The observed $\mathrm{C}^{+}$and $\mathrm{C}^{18} \mathrm{O}$ column densities transformed to total $\mathrm{H}$ column densities using standard gas phase abundance ratios $\left(X\left(\mathrm{C}^{+}\right)=1.5 \times 10^{-4}\right.$ and $X\left(\mathrm{C}^{18} \mathrm{O}\right)=1 \times 10^{-7}$; Cardelli et al. 1996; Tielens 2005) are $7 \times 10^{22}$ and $1.3 \times 10^{23} \mathrm{~cm}^{-2}$, respectively (Hogerheijde et al. 1995; Ossenkopf et al. 2013). The density in the PDR has been obtained from modeling molecular emission lines of $10^{5} \mathrm{~cm}^{-3}$ (Simon et al. 1997). Slightly lower densities have been obtained from an analysis of the PDR cooling lines and from the observed stratification of the bar adopting standard dust properties (Tielens et al. 1993), but we deem those more uncertain. Adopting the $\mathrm{C}^{+}$-derived $\mathrm{H}$-column density as appropriate for the atomic zone in the PDR, and a density of $1 \times 10^{5} \mathrm{~cm}^{-3}$, we derive a length-scale of $0.23 \mathrm{pc}$ for the line of sight. For comparison, deriving the EM associated with the ionization bar from the observed $\mathrm{H} \alpha$ intensity (Figure 4 in Wen \& O'dell 1995) and the density derived from the [S II] lines $\left(2.5 \times 10^{3} \mathrm{~cm}^{-3}\right.$, Sánchez et al. 2007), we also arrive to a line-of-sight extent of the (ionized) 

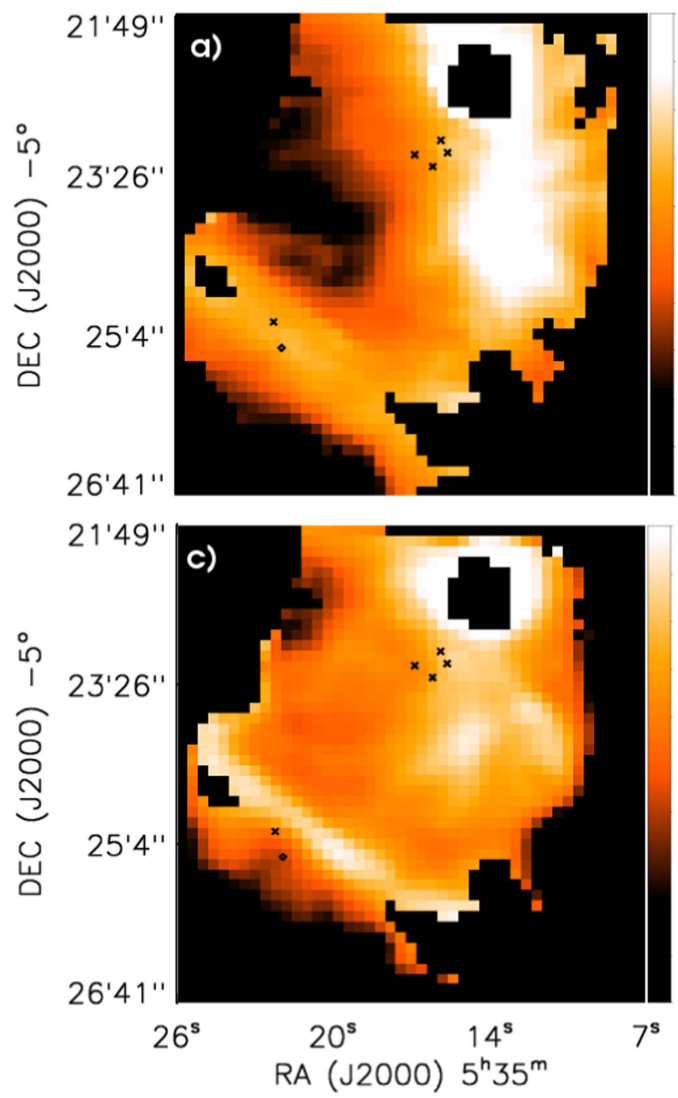
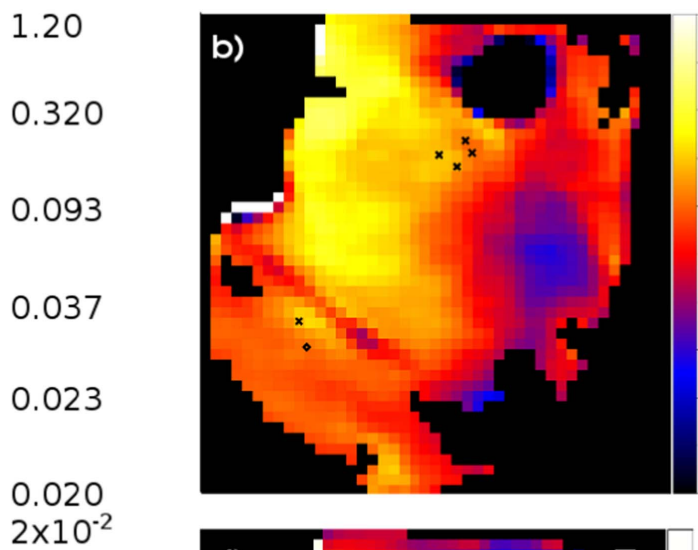

50

44

38

32

26

20

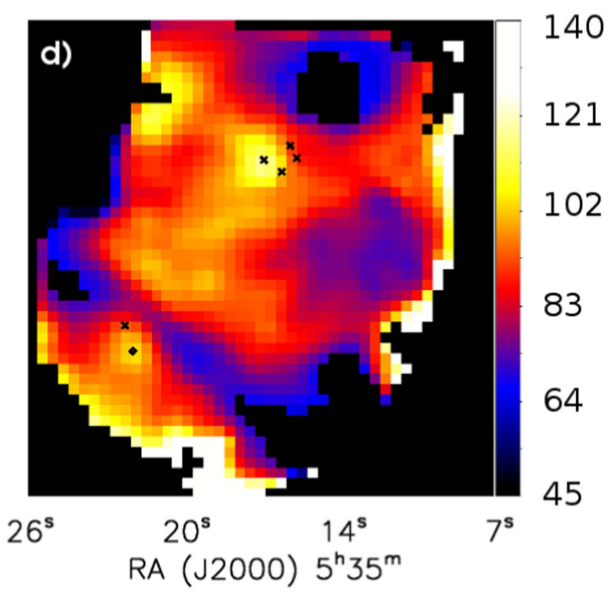

Figure 8. Temperature and optical depth maps of the two dust components revealed by the SED modeling. (a) Optical depth, $\tau_{\text {cold }}$, for the cold component. (b) Temperature map for the cold component. (c) Optical depth, $\tau_{\text {warm }}$, for the warm component. (d) Temperature map for the warm component.

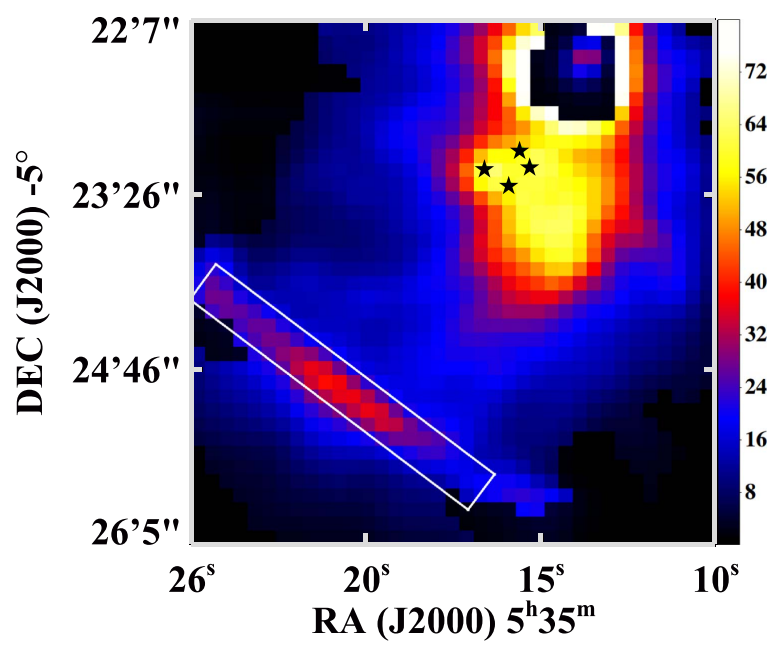

Figure 9. Total infrared luminosity from the blackbody fits; we extracted the luminosity in the aperture marked in white.

bar of $0.3 \mathrm{pc}$. Note, however, that there is a large column density of foreground ionized gas in the same direction.

In summary, adopting the Marconi et al. (1998) value for $G_{0}$ measured in the ionization front, we arrive at $l_{\mathrm{LOS}}=0.28 \mathrm{pc}$. This then implies that the actual distance from the Trapezium stars to the Orion bar is $0.33-0.45 \mathrm{pc}$ - depending on the adopted luminosity - rather than the projected distance of $0.23 \mathrm{pc}$. Finally, from the observed width of the [O I] $6300 \AA$ structure $(0.02 \mathrm{pc})$-much larger than the expected width of an ionization front $\left(10^{-3} \mathrm{pc}\right.$ Tielens 2005) - and the derived length-scale of the Orion bar, we arrive at an inclination of the Orion bar of $4^{\circ}$ with respect to the line of sight.

\subsection{The Swept-up Shell}

In Section 3.2, we identified the extent of the Orion bar on the sky as the location of the shock front, some $0.1 \mathrm{pc}$ from the ionization front. Most of the gas in this swept-up shell will be molecular and in pressure equilibrium with the atomic gas $\left(n=10^{5} \mathrm{~cm}^{-1}, T=500 \mathrm{~K}\right.$; Simon et al. 1997; Allers et al. 2005 ) in the PDR. Adopting a temperature of $50 \mathrm{~K}$ for the molecular gas, we then arrive at a density of $10^{6} \mathrm{~cm}^{-3}$. The radial column density of the bar is then $N_{\mathrm{H}} \simeq 3 \times 10^{23} \mathrm{~cm}^{-2}$ and the total mass of swept-up material is $6 \times 10^{3} M_{\odot}$. Because the mass of ionized gas is very small $\left(\simeq 10 M_{\odot}\right)$, we can infer an average density of $3 \times 10^{4} \mathrm{~cm}^{-3}$ before the star turned on; a typical density for a massive molecular cloud core (e.g., Rathborne et al. 2006; Liu et al. 2014). Comparing this density with that of the ionized gas, we arrive at a shock velocity of $v_{s} \simeq \sqrt{n_{o} / n_{e}} C_{s} \simeq 3 \mathrm{~km} \mathrm{~s}^{-1}$ with $C_{s} \approx 10 \mathrm{~km} \mathrm{~s}^{-1}$, the sound velocity in the ionized gas, and $n_{o}$ and $n_{e}$ the densities of the neutral and ionized material, respectively (Tielens 2005). Coupled with the location of the shell, we infer an age of $10^{5}$ years for $\theta^{1}$ Ori $\mathrm{C}$ and the formation of the $\mathrm{H}$ II region. This should be considered an upper limit because the shock may have decelerated with time. Using the size of a "measurable" $\mathrm{H}$ II region to infer the age of the ionizing star should be considered as an order of magnitude estimation, since this heavily depends on the structure and density 
distribution of the natal environment as well as the viewing angle of the observed system. Our value, however, is similar to that estimated by Hillenbrand (1997) for the age of low-mass stars in the region and much less than that of Simón-Díaz et al. (2006; $2.5 \pm 0.5 \mathrm{Myr})$.

\subsection{Dust Extinction}

In this section, we determine the dust opacity in the ionized gas and PDR from the observations. The results show that the UV and IR opacities are about an order of magnitude less than for the diffuse ISM. In Section 5.3, we look at the implications.

\subsubsection{The IR Extinction in the H II Region}

We can estimate the IR extinction properties of the gas in the ionized gas by comparing our fits with the radio emission measure from Dicker et al. (2009) and the electron density. From the warm dust maps, we have selected a region where the higher temperature and morphology indicates that it is associated with the ionized gas (white square in Figure 3). At this position, the measured $19.7 \mu \mathrm{m}$ optical depths is $0.025 \pm 0.003$, while the emission measure-estimated from the published emission measure map of Dicker et al. (2009) is $7 \pm 0.5 \times 10^{6} \mathrm{~cm}^{-6} \mathrm{pc}$. With the measured electron densities $\left(2500 \pm 500 \mathrm{~cm}^{-3}\right)$, we arrive at a column density of $8 \pm 2 \times 10^{21} \mathrm{~cm}^{-2}$. This then translates into $19.7 \mu \mathrm{m}$ dust opacities of $3.1 \pm 0.9 \times 10^{-24} \mathrm{~cm}^{2} / \mathrm{H}$-atom. This value is a factor of about 10 less than the measurements by Chiar \& Tielens (2006) toward Cyg OB 2 and about a factor of four less than in typical models for interstellar dust $\left(1.3\right.$ to $1.6 \times 10^{-23}$ $\mathrm{cm}^{2} / \mathrm{H}$-atom; Draine 2003).

\subsubsection{The UV Extinction in the H II Region}

In the H II region, dust grains and hydrogen compete for the absorption of stellar radiation, but Ly $\alpha$ will be (locally) absorbed by the dust. As in our previous study (Salgado et al. 2012), we can estimate the UV extinction produced by dust in the ionized region. The total IR luminosity associated with the ionization bar is measured to be $2.6 \times 10^{3} L_{\odot}$ by integrating our modified blackbody fits in this part of the $\mathrm{H}$ II region. From the emission measure image presented by Dicker et al. (2009), we estimate an average EM of $6 \times 10^{6} \mathrm{~cm}^{-6} \mathrm{pc}$, which corresponds to a Ly $\alpha$ luminosity of $1.8 \times 10^{3} L_{\odot}$. Hence, the dust heating due to absorption of star light is small in the ionized bar $\left(800 L_{\odot}\right)$. From the geometry derived in Section 3.2, we estimate that the fraction of the stellar luminosity available corresponds to $A_{\mathrm{abs}} / 4 \pi d^{2}$. We use $A_{\mathrm{abs}}=0.09 \mathrm{pc}^{2}$, which goes with an actual distance of $0.45 \mathrm{pc}$ and a luminosity of $2.7 \times 10^{5} L_{\odot}$ to arrive at the dust UV optical depth, $\tau_{\mathrm{UV}}$, of $8 \times 10^{-2}$. With the width of this region $(0.05 \mathrm{pc})$ and a density of $2.5 \times 10^{3} \mathrm{~cm}^{-3}$ (Wen \& O'dell 1995), this results in a UV dust opacity of $2 \times 10^{-22} \mathrm{~cm}^{2} / \mathrm{H}$-atom. These values can be compared to the calculated UV opacity of dust in the diffuse ISM, which-for an $R_{V}$ of 5.5-ranges from 1 to $1.5 \times 10^{-21}$ $\mathrm{cm}^{2} / \mathrm{H}$-atom over the wavelength range of $2200-912 \AA$ (Weingartner \& Draine 2001; Draine 2003). Similar values are actually obtained for sight lines in the diffuse ISM characterized by $R_{V}=3.1$. Hence, the UV opacity of dust in the Orion $\mathrm{H}$ II region is a factor of $\simeq 5$ less than in the diffuse ISM.

\subsubsection{IR Extinction in the PDR}

The $19.7 \mu \mathrm{m}$ optical depth of the warm dust in the Orion bar PDR is measured to be 0.2 (Figure 8). Taking the H-column density derived from the CII observations $\left(7 \times 10^{22} \mathrm{~cm}^{2}\right.$; Ossenkopf et al. (2013); see Section 4.1), we arrive at a $19.7 \mu \mathrm{m}$ extinction per $\mathrm{H}$-atom of $2.9 \times 10^{-24} \mathrm{~cm}^{2} / \mathrm{H}$-atom. These values are a factor of 10 less than the values measured toward Cyg OB2 $\left(3.7 \times 10^{-23} \mathrm{~cm}^{2} / \mathrm{H}\right.$-atom Chiar \& Tielens 2006) and a factor of five less than the models from Draine (2003; 1.3 to $1.6 \times 10^{-23} \mathrm{~cm}^{2} / \mathrm{H}$-atom, depending on the value for $R_{V}$ ).

\subsubsection{The Penetration of UV Photons in the PDR}

Since PAHs are excited by FUV photons, the decrease in PAH emission traces the dust grain extinction through the PDR. The emission of PAHs at a distance $d$ is given by

$$
F_{\mathrm{PAH}}(d)=F_{\mathrm{PAH}}(\text { peak }) \times e^{-\tau_{\mathrm{UV}}(\text { radial })},
$$

with $\tau_{\mathrm{UV}}($ radial $)=N_{d}^{T} \times \sigma_{\mathrm{UV}}$ the radial optical depth. The $\mathrm{PAH}$ emission decreases by a factor of eight over a length of $30^{\prime \prime}(0.06 \mathrm{pc})$ from the peak emission. With a density of $10^{5} \mathrm{~cm}^{-3}$, this translates into a UV opacity of $1.6 \times 10^{-22}$ $\mathrm{cm}^{2} / \mathrm{H}$-atom. Hence, as for the dust in the ionized gas, the dust in the PDR is characterized by a UV opacity, which is about a factor of 10 less than for dust in the diffuse ISM.

\section{DISCUSSION}

\subsection{The Dust Temperature and Lyo Heating}

It has been long suspected that trapped $\operatorname{Ly} \alpha$ photons can be a major source of dust heating in H II regions (e.g., Wright 1973; Garay et al. 1993; Smith et al. 1999). As the discussion in Section 4.3.2 demonstrates, Ly $\alpha$ photons are very important for the heating of dust in the Orion Nebula. Here, we will compare the derived temperatures of the warm dust component. The total heating of the dust depends on the distance to the central star, the radial extinction of UV photons toward the star, and the number of trapped Ly $\alpha$ photons. These two sources of heating lead to a different distance dependence for the temperature. Because of resonant scattering, Ly $\alpha$ photons are absorbed on the spot and the contribution to the heating rate is

$$
\Gamma_{\mathrm{Ly} \alpha}=n_{e} n_{\mathrm{H}} f \alpha_{\mathrm{H}}^{(2)} h \nu_{\alpha} / n_{d},
$$

where $n_{e}$ is the electron density, $n_{\mathrm{H}}$ is the hydrogen density, $n_{d}$ is the dust density, $h \nu_{\alpha}$ is the energy of the Ly $\alpha$ photon $(10.2 \mathrm{eV}), f=0.7$ is the fraction of recombination to levels higher than two that lead to emission of a Ly $\alpha$ photon, and $\alpha_{\mathrm{H}}^{(2)}$ is the recombination coefficient of hydrogen (case B). In addition, there is the direct stellar radiation,

$$
\Gamma_{d}(a, r)=\pi a^{2} \frac{L_{\star}(\mathrm{UV})}{4 \pi r^{2}} e^{-\tau_{\text {radial }}} Q_{\text {abs }}(\mathrm{UV})+\Gamma_{\mathrm{Ly} \alpha}
$$

where $\tau_{\text {radial }}$ is the UV optical depth from the star to a point $r$ and $a$ is the radius of a spherical grain. Note that the stellar UV luminosity in terms of $G_{0}$ is $L_{\star}(\mathrm{UV}) e^{-\tau_{\text {radial }}} / 4 \pi R_{s}^{2}$. The dust temperature can be obtained by equating the heating with the wavelength integrated emission of a dust grain. The emission can be approximated well by

$$
\Gamma_{\mathrm{em}}=4 \pi a^{2} Q_{0} \sigma T_{d}^{6},
$$




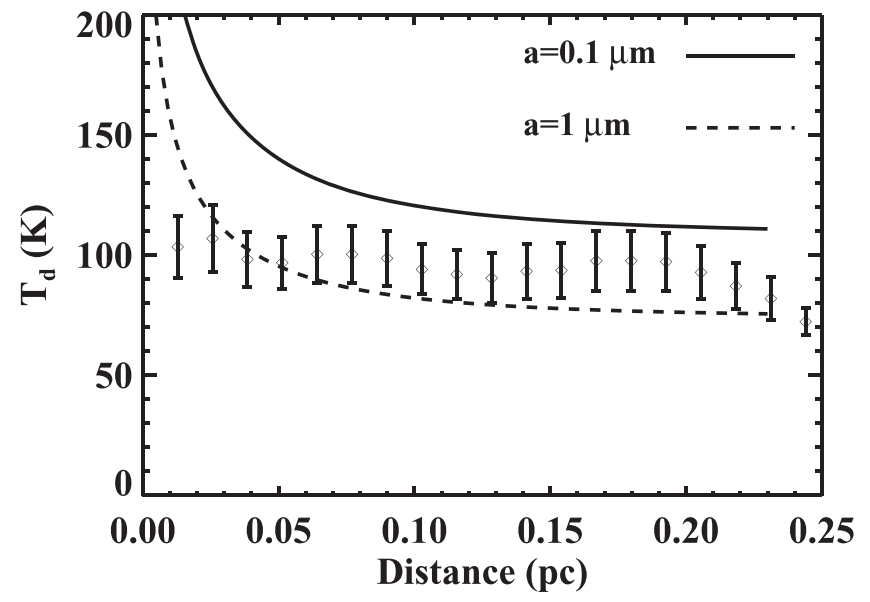

Figure 10. Temperature as a function of distance for different dust grain size: $0.1 \mu \mathrm{m}$ (solid line) and $1 \mu \mathrm{m}$ (dashed line). Symbols represent derived dust temperatures of the warm component.

where $\sigma$ is the Stefan-Boltzmann constant and for the constant $Q_{0}$, we adopt the properties of silicates $\left[Q_{0}=1.25 \times 10^{-5}(a /\right.$ $0.1 \mu \mathrm{m})^{0.06}$, Tielens 2005]. The dust temperature is then given by

$$
T_{d}^{6}=\frac{Q_{\mathrm{abs}}(\mathrm{UV}) G_{0}}{4 Q_{0} \sigma}\left[\frac{3 h \nu_{\alpha} f}{\tau_{d} \overline{h \nu}}+\left(\frac{R_{s}}{r}\right)^{2}\right],
$$

where $\overline{h \nu}$ is the average photon energy per ionizing photon $\left(L_{\star} / N_{\text {ion }} \simeq 49 \mathrm{eV}, \quad\right.$ for the parameters of $\theta^{1}$ Ori C), $R_{s}=0.23 \mathrm{pc}$ is the Strömgren radius and $\tau_{d}=0.08$ is the dust optical depth. We have adopted $G_{0}=2.6 \times 10^{4}$ (Section 4.1$), Q_{\mathrm{abs}}(\mathrm{UV}) \approx 1$. We emphasized that all these parameters-except for the detailed absorption/emission properties of the dust-are directly derived from observations. Note that the calculated dust temperature depends on the ratio of the UV absorption to the IR emission cross section. While we have derived much reduced dust opacities in Section 4.3, the ratio of the two is actually similar to what we have adopted here.

The results are compared to the observed temperature in Figure 10. The calculated temperatures show the expected characteristic of $\operatorname{Ly} \alpha$ heating; i.e., constant dust temperature throughout the ionized gas. Heating by the photons from the central star does not start to dominate the dust temperature until $r \lesssim 0.25 R_{s}$. We note that if we exclude Ly $\alpha$ heating, the geometric dilution of the radiation field leads to a noticeable decrease in the dust temperature throughout the region. This is not present in the data, again illustrating the importance of Ly $\alpha$ heating of the dust. The absence of an upturn in the observed temperature at small radii likely reflects the absence of dust close to $\theta^{1}$ Ori $\mathrm{C}$ because the stellar wind may have created a dust-free cavity. Finally, in order to reproduce the observed temperatures, dust grains with sizes between 0.1 and $1 \mu \mathrm{m}$ are required. This value is somewhat large compared to the dust in the diffuse ISM. We discuss this further in Section 5.2.

\subsection{Properties of the Dust}

We have determined opacities in the UV and IR - in units of $\mathrm{cm}^{2} / \mathrm{H}$-atom-both for dust in the ionization bar and in the PDR (see Section 4.3). As emphasized before, the derived UV and IR opacities are reduced by a factor of 5-10 as compared to dust in the diffuse ISM. Recently, the Planck Collaboration et al. (2014b) reported maps of the spectral index of the whole sky including the Orion Nebula, where they report a value of $\beta=1.6$. Using the Planck value $(\beta=1.6)$, we obtain differences in the temperatures of dust for the cold and warm components of $\sim 7 \%$ and $\sim 5 \%$, respectively. The small difference in temperature further reduces the column densities, and thus the opacities, by a factor of approximately three. We attribute the decreased opacity values to the effects of coagulation. Indeed, theoretical studies show that coagulation can greatly reduce the extinction per unit mass both in the UV and the IR, if the grains can grow to very large sizes (Ossenkopf \& Henning 1994; Ormel et al. 2011). A similar result was obtained by Lombardi et al. (2014) analyzing the near-infrared extinction and submillimeter Planck maps for the Orion A and B clouds. Detailed models show that a factor of 10 reduction in the opacity is reached after a time, $t \simeq 100 \tau_{0}$, where $\tau_{0}$ is the collision timescale at the onset of coagulation,

$$
\begin{aligned}
\tau_{0}= & \left(n_{d} \Delta v \sigma_{d}\right)^{-1} \simeq 8.5 \times 10^{4} \text { years }\left(\frac{a_{0}}{0.1 \mu \mathrm{m}}\right)^{1 / 2} \\
& \times\left(\frac{n}{10^{5} \mathrm{~cm}^{-3}}\right)^{-3 / 4}\left(\frac{T}{10 \mathrm{~K}}\right)^{-1 / 4},
\end{aligned}
$$

where $n_{d}, \Delta v$, and $\sigma_{d}$ are the number density, relative velocity, and cross section of the grains (Ormel et al. 2009). In the righthand side of this equation, an initially normal dust-to-gas ratio was adopted and $a_{0}, n$, and $T$ are the size of the monomer, gas density, and temperature, respectively. At this point $\left(t \simeq 100 \tau_{0}\right)$, the calculated porosity of the aggregates is $\simeq 0.3$ and the aggregate size is $\sim 10 \mu \mathrm{m}$. From this timescale, we infer that the large grain size must reflect coagulation before the formation of $\theta^{1}$ Ori $\mathrm{C}$, the $\mathrm{H}$ II region, and the PDR; i.e., during the cold dark cloud phase of Orion A. During this phase, coagulation is much assisted by the presence of ice mantles. However, when the dust temperature reaches some $75 \mathrm{~K}$, the ice will sublimate on a timescale of $10^{4}$ years, but we surmise that ice sublimation associated with such gentle thermal processing will not affect the structure of the dust aggregates. Once the dust leaves the shielded environment of the PDR and enters the H II region, further processing may occur. Sputtering will have little effect at $T=10^{4} \mathrm{~K}$, but grain-grain collisions, even at $\simeq 5 \mathrm{~m} \mathrm{~s}^{-1}$, may lead to fragmentation (Wada et al. 2013; Krijt et al. 2015). However, our results suggest that this processing takes place on a timescale that is long compared to the evolution of the $\mathrm{HII}$ region. Large dust grains seem to be a common characteristic of dense cloud cores as evidenced from near-infrared scattered light images (e.g., Pagani et al. 2010; Steinacker et al. 2010). Likewise, analysis of the IR emission from the H II region, IC 434, also inferred the presence of large dust aggregates in the champagne flow from the dark cloud, L1630 Ochsendorf et al. (2014a).

\subsection{Dust in H II Regions}

There has been a long history of infrared studies of dust in H II regions (e.g., Ney et al. 1973; Harper 1974). Here, we will place our analysis of the Orion H II region in context with a 
recent study of the W3(A) H II region and the results from the GLIMPSE survey.

In a previous study, Salgado et al. (2012) analyzed the dust emission in W3(A) using the same mid-infrared FORCAST filters. Like Orion, W3(A) is a young, compact $\mathrm{H}$ II region and is powered by a similar ionizing star. The ionized gas emission in the Orion nebula, as traced by $\mathrm{H} \alpha$, is high close to the Trapezium stars, then it slowly decreases up to $0.18 \mathrm{pc}$ where it sharply increases, while at larger distances it sharply decreases again (Figure 4). Analysis of the optical emission morphology and spectra of the $\mathrm{H}$ II region reveals that the ionized gas is distributed in a thin shell $(0.02 \mathrm{pc})$ bounded at the outside by the ionization front (Wen \& O'dell 1995). The ionized gas (as traced by free-free emission at $2 \mathrm{~cm}$ ) in $\mathrm{W} 3(\mathrm{~A})$ has a similar structure, but the ionized gas shell is much thicker $(\simeq 0.1 \mathrm{pc})$.

When comparing the dust emission of Orion with W3 (A), we also see some similarities in the spatial distribution. In Orion, the $19 \mu \mathrm{m}$ emission shows a component coinciding with the Orion bar and one that is associated with dust in the ionized gas (Figure 3; Section 3.2). The other IR dust/PAH tracers only peak in the Orion bar PDR. For W3(A), the $19.7 \mu \mathrm{m}$ emission traces dust well mixed in with the ionized gas shell. As for Orion, at other wavelengths, the dust (and PAH) emission clearly peak in the PDR surrounding the ionized shell. Hence, for both regions, the $19.7 \mu \mathrm{m}$ flux originates from dust mixed in with the ionized gas. We note that unlike Orion, the $19.7 \mu \mathrm{m}$ map of W3(A) does not show a secondary peak at the PDR (Salgado et al. 2012). In the PDR, the dust is exclusively heated by stellar photons and this difference in $19.7 \mu \mathrm{m}$ morphology merely reflects the smaller scale size and concomitantly higher dust temperature for Orion as compared to $\mathrm{W} 3(\mathrm{~A})$.

The heating of the dust is, however, different for these two $\mathrm{H}$ II regions. In Orion, resonantly scattered Ly $\alpha$ photons contribute some two-thirds of the dust heating in the ionized gas; the remainder is due to absorption of stellar FUV photons. In contrast, for W3(A), dust heating is dominated by stellar photons with only a minor contribution from Ly $\alpha$. Our analysis shows that this reflects the very low optical depth of dust in the ionized gas in Orion ( $\tau_{\mathrm{UV}} \simeq 0.08$ ). For W3(A), the UV optical depth was measured to be $\simeq 1$ and consequently stellar heating is much more important (see Equation (2)). To phrase it differently, irrespective of the dust properties, all the Ly $\alpha$ energy will eventually wind up in the dust. In contrast, the absorption of stellar photons depends strongly on the dust opacity per H-atom and, for these two regions, the derived UV dust opacities (per H-atom) differ by almost a factor of 100 .

This difference in dust opacity per $\mathrm{H}$-atom must reflect a difference in evolution between these two regions. Both stars formed in dense cores where coagulation had likely reached a steady state. Both H II regions are also very young: W3(A) is slightly larger but Orion is already optically visible. Morphologically speaking, for W3(A), the shell structure likely betrays the importance of radiation pressure on the ionized gas and the dust, though we recognize that theoretical models predict a much shallower rise in the shell density than observed (Draine 2011). In contrast, for Orion, the $\mathrm{H}$ II region has broken out of the molecular cloud and has created a champagne flow (Güdel et al. 2008) and the dust is dragged along (Ochsendorf et al. 2014a). The difference in dust properties might therefore originate in, or relate to, the champagne flow phase in the evolution of compact $\mathrm{H}$ II regions that W3(A) has not yet

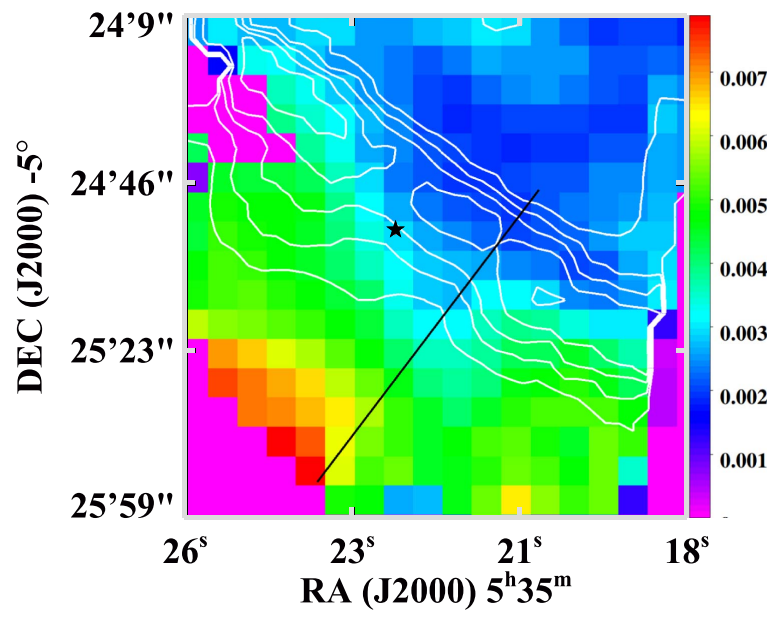

Figure 11. Photoelectric heating efficiency map. The star marks the position of $\theta^{2}$ Ori A and the line corresponds to the cross-cut for regions in Figure 12. The cross-cut extends from the surface of the PDR to deep in the molecular cloud, with a position angle of $143^{\circ}$ measured counter-clockwise from the north. Overlaid in white is the contour map of [O I], the main cooling line. The photoelectric heating efficiency increases with distance from the trapezium stars.

entered in. Therefore, it possibly reflects a size-sorting of the dust due to radiation pressure during the blister phase (Ochsendorf \& Tielens 2015).

The Spitzer/GLIMPSE survey has revealed some 6000 bubble $\mathrm{H}$ II regions in the Milky Way. These are invariably characterized by $24 \mu \mathrm{m}$ emission associated with dust inside the bubble while emission at $8 \mu \mathrm{m}$ and at far-infrared wavelength traces the PDR surrounding the $\mathrm{H}$ II region (Churchwell et al. 2006, 2007; Deharveng et al. 2010). The morphology of the compact H II regions, Orion and W3(A), is very similar where dust in the ionized gas is visible at $19.7 \mu \mathrm{m}$ while the PDR is traced in the PAH and colder dust emission. As the SED of the dust in the ionized gas peaks around $20 \mu \mathrm{m}$ in all cases, the dust must have very similar temperatures, suggesting that Ly $\alpha$ heating may dominate dust heating in H II regions in general. Alternatively, the $24 \mu \mathrm{m}$ emission in these bubbles may trace a dust wave where the radiation pressure of the stellar light stops onrushing dust in a champagne flow and forces it into an arc-like structure around the star (Ochsendorf et al. 2014a, 2014b). Because the stellar properties are very similar, the dust wave may form at very similar distances, resulting in very similar temperatures.

\subsection{Cooling Lines and Photoelectric Heating Efficiency}

In dense PDRs, gas cooling is dominated by emission of forbidden transition lines of [O I] at $63 \mu \mathrm{m}$ and [C II] at $157 \mu \mathrm{m}$ (Hollenbach \& Tielens 1999). Observations of the gas cooling lines in the Orion bar have been discussed extensively by Bernard-Salas et al. (2012). Here we reanalyzed the data, including the mid-infrared contribution to the SED and the total IR emission of this region. In PDRs, the dominant heating process is due to the photoelectric effect in PAHs and small grains (de Jong et al. 1980; Bakes \& Tielens 1994). The efficiency of the process can be measured directly from the ratio between the main gas cooling lines and the total energy absorbed by PAHs and dust grains. 

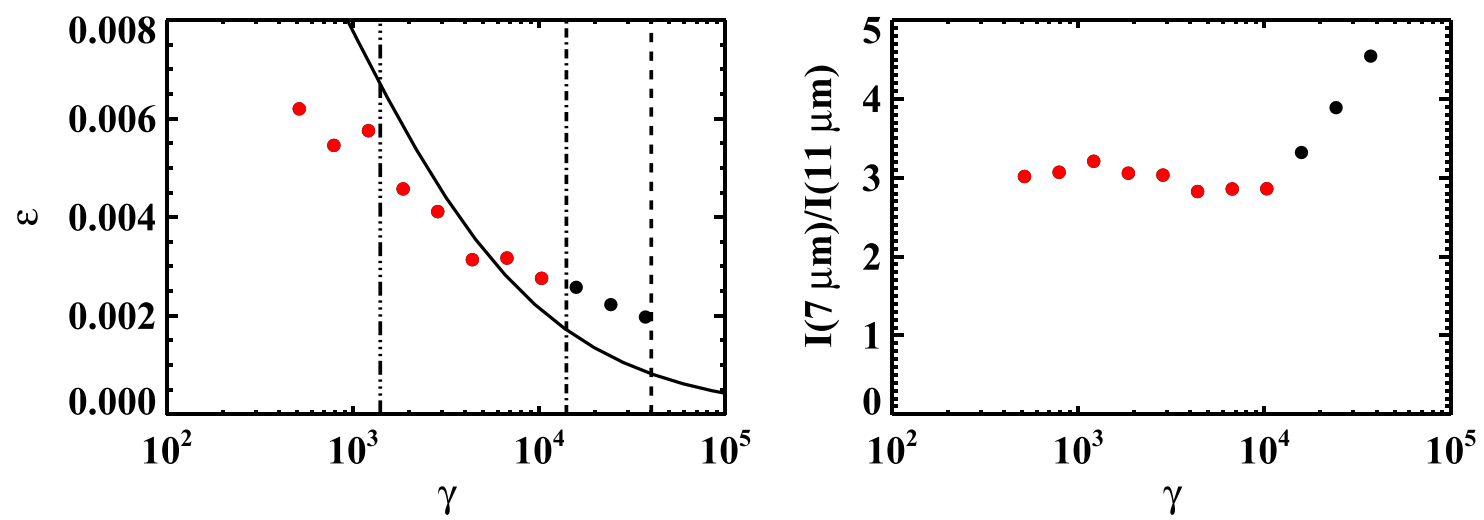

Figure 12. Photoelectric heating efficiency as a function of ionization parameter $\left(G_{0} \sqrt{T} / n_{e}\right)$ along the cross-cut through the Orion bar (Figure 11). The dashed line marks the position of the ionization front, the dotted-dashed line the position of $\mathrm{H}_{2}$ peak and the dotted-dotted-dashed the end of swept-up gas; the solid line is the model for photoelectric heating from Bakes \& Tielens (1994) scaled down by a factor of three. $G_{0}$ was estimated from the incident radiation field attenuated in the bar. The temperature $(T=500 \mathrm{~K})$ and electron density $\left(n_{e}=15 \mathrm{~cm}^{-3}\right)$ were assumed to be constant (see the text for details). Black dots refer to warm $(T \simeq 500 \mathrm{~K})$ gas traced by the pure rotational lines of $\mathrm{H}_{2}$. The red dots are cooler molecular gas $(T \simeq 50-100 \mathrm{~K})$ traced by CO.

We used the PACS [O I] at 63 and $145 \mu \mathrm{m}$, and the [C II] at $157 \mu \mathrm{m}$ lines to determine the photoelectric heating efficiency, i.e., the ratio between $[\mathrm{OI}]_{63}+[\mathrm{C} \mathrm{II}]_{157}+[\mathrm{OI}]_{145}$ and the total IR emission derived from the blackbody fits (Section 3.3). Typical flux values for the lines are $7 \times 10^{-2}$ and $6 \times 10^{-3} \mathrm{erg} \mathrm{s}^{-1} \mathrm{~cm}^{-2} \mathrm{sr}^{-1}$ for the [O I] at $63 \mu \mathrm{m}$ and $145 \mu \mathrm{m}$, respectively. For the [C II] line, a typical value is $8 \times 10^{-3} \mathrm{erg} \mathrm{s}^{-1} \mathrm{~cm}^{-2} \mathrm{sr}^{-1}$. In the Orion bar, we find an efficiency of $2 \times 10^{-3}$, which increases further into the PDR and can reach values as high as $7 \times 10^{-3}$ (see Figure 11). The values found here are similar to those derived from Table 3 in Herrmann et al. (1997) and for M17 (Meixner et al. 1992). In this analysis, we assume that the far-infrared dust continuum emission is a good measure for the UV flux locally absorbed. That is a good assumption at the surface of the PDR, where FUV photons dominate. However, deeper in, the stellar UV flux is attenuated and absorption of mid-infrared photons produced by warm dust in the surface layers of the PDR becomes an important dust heating source. The effect of this is that the actual increase of the heating efficiency (defined as gas heating over absorbed FUV flux; Tielens \& Hollenbach 1985) increases even more than the factor of two indicated above. This increase in the heating efficiency with depth in the PDR is a standard feature of photoelectric heating models. As the UV field decreases, the PAHs and very small dust grains become less positively charged and the heating efficiency increases (Bakes \& Tielens 1994).

In Figure 12, we show the photoelectric efficiency as a function of the ionization parameter, $\gamma=G_{0} \sqrt{T} / n_{e}$ along a line perpendicular to the Orion bar surface, which avoids - as best as possible - the clumps present in the region. In this analysis, we assume that the region is homogeneous in temperature $(T=500 \mathrm{~K}$; Parmar et al. 1991; Allers et al. 2005) and an electron density $\left(n_{e}=X_{e} n_{\mathrm{H}}\right)$ with an electron abundance equal to the gas phase carbon abundance $\left(X_{e}=1.5 \times 10^{-4}\right.$; Cardelli et al. 1996) and $n_{\mathrm{H}}=10^{5} \mathrm{~cm}^{-3}$ (Simon et al. 1997). The variation in the ionization parameter is then largely due to variation in the penetrating UV field, which we approximate by the incident radiation field $\left(G_{0}=2.6 \times 10^{4}\right.$ in Habing units, Section 4.1) extincted by $\tau_{\mathrm{UV}}=8\left(d / 30^{\prime \prime}\right)$ (Section 4.3.4). The figure illustrates well that as the ionization parameter increases, the heating efficiency decreases. A similar trend was also noted by Okada et al. (2013) in their analysis of a sample of six PDRs.
However, because their data set is very inhomogeneous and the ionization parameters are difficult to determine in an absolute sense, the trend is less clear. In this study, we focus on a single region where variations in the ionization parameter are driven by the drop of the radiation field and a clear trend is revealed. We do note, however, that the absolute scale of the ionization parameter does depend on the adopted temperature and density. It is reasonable to assume that the region is in pressure equilibrium and hence the ionization parameter would scale as $G_{0} / \sqrt{T}$. We have indicated points beyond the peak of the $\mathrm{H}_{2}$ emission where this variation in temperature would become relevant as red in Figure 12. The ionization parameter at these locations might have been underestimated by up to a factor of $\simeq 2.5$ (i.e., $\sqrt{T_{\mathrm{H}_{2}} / T_{\mathrm{CO}}}=\sqrt{500 / 75}$ ).

We recognize the general increase in the heating efficiency as predicted by model studies (Bakes \& Tielens 1994; Weingartner \& Draine 2001). However, the observed heating efficiency differs from that expected from models. The theoretical models were developed for the diffuse ISM, where observed heating efficiencies are higher (Wolfire et al. 1995; Hollenbach \& Tielens 1999). This difference in heating efficiency may just reflect a difference in dust properties; i.e., a lower abundance of PAHs and very small grains in the Orion bar PDR (Section 5.2).

The photoelectric heating efficiency is expected to be linked to the degree of ionization of the PAHs and very small grains. For PAHs, the degree of ionization can be traced by the ratio between the $7.7 \mu \mathrm{m}$ and the $11.3 \mu \mathrm{m}$ features as the $7.7 \mu \mathrm{m}$ feature is emitted by ionized PAH and the $11.3 \mu \mathrm{m}$ by neutral PAHs (DeFrees et al. 1993; Langhoff 1996; Allamandola et al. 1999). Following Galliano et al. (2008), we used the ISOCAMCVF data to get values for the ionized to neutral ratio of PAHs (Figure 12). As expected, there is a clear increase in the fraction of PAH cations from the $\mathrm{H}_{2}$ peak to the front of the PDR, in good agreement with the trend found by Galliano et al. (2008). However, we notice that the PAH ratio reaches a plateau starting at the $\mathrm{H}_{2}$ peak until the end of the dense bar (which we associate with the swept-up shell). Perhaps, this reflects the influence of anions deep in the PDR.

As noted in Section 3.2, both the cooling line and the dust emission display a clumpy distribution. We also see variations of the photoelectric heating efficiency parallel to the Orion bar surface. However, the clumpy structure apparent in the cooling 
line maps is not seen in the photoelectric heating map (Figure 11). It seems that the increased density associated with clumps does not translate to an increased photoelectric heating efficiency. These observations illustrate that further detailed studies of the cooling lines and dust emission coupled with density probes (such as molecular tracers) are needed to probe the relationship between gas cooling and the $\mathrm{PAH} / \mathrm{dust}$ components responsible for the heating.

\section{SUMMARY AND CONCLUSIONS}

We analyzed the infrared emission in the Orion star-forming region in six bands observed with the FORCAST instrument on board SOFIA. We complemented the data set with public published photometry and spectroscopy ranging from optical to far-infrared wavelengths. The FORCAST images show a complex structure and we recognize emission arising in the Orion bar PDR, the BN/KL object, and the Ney-Allen nebula. Our analysis shows that the Herschel/PACS far-infrared emission is associated with the cold molecular cloud. The mid-infrared emission is not spatially correlated with the farinfrared emission and must be produced in the PDR and, partially, in the $\mathrm{H}$ II region.

We constructed SEDs after convolving the FORCAST images to the PACS $160 \mu \mathrm{m}$ resolution. By fitting the SEDs with two modified blackbodies, we derived dust temperatures and column densities for dust associated with the molecular cloud and the PDR/H II region. Most of the dust mass is in the cold dust component, which is associated with the background cold and dense molecular cloud. The luminosity, on the other hand, is dominated by the warm dust component associated with the PDR/H II region. We obtained the total infrared luminosity by integrating our blackbody fits over wavelengths between 1 and $1000 \mu \mathrm{m}$. The results of the fits were used to obtain the geometry of the Orion bar PDR. We measured a depth along the line of sight of $0.28 \mathrm{pc}$ and an inclination angle of $4^{\circ}$.

We have determined that the Orion bar extends from the ionization front located at $0.23 \mathrm{pc}$ from $\theta^{1}$ Ori $\mathrm{C}$ up to the shock front located at about $0.1 \mathrm{pc}$ from the ionization front. The amount of mass swept by the expansion of the $\mathrm{H}$ II region is $6 \times 10^{3} M_{\odot}$ and we estimate an age of $\simeq 10^{5}$ years for the $\mathrm{H}$ II region and $\theta^{1}$ Ori $\mathrm{C}$. This age is much less than that derived by stellar evolutionary models (2.5 Myr, Simón-Díaz et al. 2006).

By comparing the $19.7 \mu \mathrm{m}$ image with ionized gas radio emission, we have determined the opacity (extinction per hydrogen atom) in the $\mathrm{H}$ II region to be a factor of five lower than models for the diffuse ISM. In addition, we have computed the UV opacity in the ionized gas by comparing the infrared emission and radio estimates. As for the infrared opacity, the UV opacity value is a factor of five lower than that of the diffuse ISM.

We measured infrared opacity in the PDR by comparing the optical depth and the density. The value obtained by this method is a factor of five less than models for the diffuse ISM. By measuring the penetration of UV photons in the PDR, as traced by the PAH emission, we derived an UV opacity a factor of 10 less than that of the ISM.

Our analysis shows that Ly $\alpha$ heating is important in Orion at distances of $r>0.25 R_{s}$. The dust temperature profile can be explained by the presence of large grains (between $0.1-1 \mu \mathrm{m}$ ).
The lower opacities as compared to ISM values and the Ly $\alpha$ heating behavior depends on the size of the dust grains. The observed values for the opacities and dust temperature in the ionized gas can be explained by the presence of large grains. We attribute the increase in size of the grains to dust coagulation during the molecular cloud phase previous to the birth of the ionizing stars.

We measure the photoelectric heating in the Orion bar from the observed fine-structure cooling lines and the IR continuum emission. The observations reveal that the photoelectric efficiency decreases toward the surface of the PDR. Theoretical models attribute this to a charging-up of the PAHs and very small grains. We estimate values for the ionization parameter as a function of depth in the Orion bar PDR. The derived heating efficiency as a function of ionization parameter agrees qualitatively well with the models but is less by about a factor of five. As the models were derived for the diffuse ISM, this quantitative difference may merely reflect a difference in PAH abundance.

We thank the FORCAST engineering team: George Gull, Justin Schoenwald, and Chuck Henderson, and the USRA Science and Mission Ops teams, and the entire SOFIA staff. F.S. would like to thank Prof. Alain Abergel and Prof. Malcolm Walmsley for useful comments and discussions during the preparation of this manuscript. This work is based on observations made with the NASA/DLR Stratospheric Observatory for Infrared Astronomy (SOFIA). SOFIA science mission operations are conducted jointly by the Universities Space Research Association, Inc. (USRA), under NASA contract NAS2-97001, and the Deutsches SOFIA Institut (DSI) under DLR contract 50 OK 0901. Financial support for FORCAST was provided by NASA through award 8500-98014 issued by USRA. Studies of interstellar PAHs and dust at Leiden Observatory are supported through advanced-ERC grant 246976 from the European Research Council, through the Spinoza premie of NWO, and through the Dutch Astrochemistry Network funded by the Dutch Science Organization, NWO. This research has made use of the SIMBAD database, operated at CDS, Strasbourg, France. This research has made use of the NASA/ IPAC Infrared Science Archive, which is operated by the Jet Propulsion Laboratory, California Institute of Technology, under contract with the National Aeronautics and Space Administration.

\section{REFERENCES}

Abergel, A., Arab, H., Compiègne, M., et al. 2010, A\&A, 518, L96 Allamandola, L. J., Hudgins, D. M., \& Sandford, S. A. 1999, ApJL, 511, L115 Allers, K. N., Jaffe, D. T., Lacy, J. H., Draine, B. T., \& Richter, M. J. 2005 , ApJ, 630, 368

Anderson, L. D., Zavagno, A., Deharveng, L., et al. 2012, A\&A, 542, A10 Arab, H., Abergel, A., Habart, E., et al. 2012, A\&A, 541, A19

Bakes, E. L. O., \& Tielens, A. G. G. M. 1994, ApJ, 427, 822 Bernard-Salas, J., Habart, E., Arab, H., et al. 2012, A\&A, 538, A37 Boersma, C., Rubin, R. H., \& Allamandola, L. J. 2012, ApJ, 753, 168 Buckle, J. V., Davis, C. J., Francesco, J. D., et al. 2012, MNRAS, 422, 521 Calzetti, D., Kennicutt, R. C., Engelbracht, C. W., et al. 2007, ApJ, 666, 870 Calzetti, D., Wu, S.-Y., Hong, S., et al. 2010, ApJ, 714, 1256 Cardelli, J. A., Clayton, G. C., \& Mathis, J. S. 1989, ApJ, 345, 245 Cardelli, J. A., Meyer, D. M., Jura, M., \& Savage, B. D. 1996, ApJ, 467, 334 Cesarsky, D., Jones, A. P., Lequeux, J., \& Verstraete, L. 2000, A\&A, 358, 708 Chiar, J. E., \& Tielens, A. G. G. M. 2006, ApJ, 637, 774

Churchwell, E., Povich, M. S., Allen, D., et al. 2006, ApJ, 649, 759 Churchwell, E., Watson, D. F., Povich, M. S., et al. 2007, ApJ, 670, 428 de Jong, T., Boland, W., \& Dalgarno, A. 1980, A\&A, 91, 68 
DeFrees, D. J., Miller, M. D., Talbi, D., Pauzat, F., \& Ellinger, Y. 1993, ApJ, 408, 530

Deharveng, L., Schuller, F., Anderson, L. D., et al. 2010, A\&A, 523, A6

Dicker, S. R., Mason, B. S., Korngut, P. M., et al. 2009, ApJ, 705, 226

Doi, T., O’Dell, C. R., \& Hartigan, P. 2004, AJ, 127, 3456

Draine, B. T. 2003, ARA\&A, 41, 241

Draine, B. T. 2011, ApJ, 732, 100

Draine, B. T., \& Li, A. 2001, ApJ, 551, 807

Draine, B. T., \& Li, A. 2007, ApJ, 657, 810

Dupac, X., Bernard, J.-P., Boudet, N., et al. 2003, A\&A, 404, L11

Everett, J. E., \& Churchwell, E. 2010, ApJ, 713, 592

Gagné, M., Oksala, M. E., Cohen, D. H., et al. 2005, ApJ, 628, 986

Galliano, F., Madden, S. C., Tielens, A. G. G. M., Peeters, E., \& Jones, A. P. 2008, ApJ, 679, 310

Garay, G., Rodriguez, L. F., Moran, J. M., \& Churchwell, E. 1993, ApJ, 418, 368

Güdel, M., Briggs, K. R., Montmerle, T., et al. 2008, Sci, 319, 309

Habing, H. J. 1968, BAN, 19, 421

Haraguchi, K., Nagayama, T., Kurita, M., Kino, M., \& Sato, S. 2012, PASJ, 64,127

Harper, D. A. 1974, ApJ, 192, 557

Henning, T. 2010, ARA\&A, 48, 21

Herrmann, F., Madden, S. C., Nikola, T., et al. 1997, ApJ, 481, 343

Herter, T. L., Adams, J. D., De Buizer, J. M., et al. 2012, ApJL, 749, L18

Herter, T. L., Vacca, W. D., Adams, J. D., et al. 2013, PASP, 125, 1393

Hillenbrand, L. A. 1997, AJ, 113, 1733

Hirsch, L., Adams, J. D., Herter, T. L., et al. 2012, ApJ, 757, 113

Hogerheijde, M. R., Jansen, D. J., \& van Dishoeck, E. F. 1995, A\&A, 294, 792

Hollenbach, D. J., \& Tielens, A. G. G. M. 1997, ARA\&A, 35, 179

Hollenbach, D. J., \& Tielens, A. G. G. M. 1999, RvMP, 71, 173

Johnstone, D., \& Bally, J. 1999, ApJL, 510, L49

Kassis, M., Adams, J. D., Campbell, M. F., et al. 2006, ApJ, 637, 823

Kraus, S., Balega, Y. Y., Berger, J.-P., et al. 2007, A\&A, 466, 649

Krijt, S., Ormel, C. W., Dominik, C., \& Tielens, A. G. G. M. 2015, A\&A, 574, A83

Kurucz, R. L. 1993, Kurucz CD-ROM (MA, Cambridge: Smithsonian Astrophysical Observatory)

Langhoff, S. R. 1996, JPhCh, 100, 2819

Lau, R. M., Herter, T. L., Morris, M. R., \& Adams, J. D. 2014, ApJ, 794, 108

Lau, R. M., Herter, T. L., Morris, M. R., Becklin, E. E., \& Adams, J. D. 2013, ApJ, 775, 37

Lee, T. A. 1968, ApJ, 152, 913

Liu, X.-L., Wang, J.-J., \& Xu, J.-L. 2014, MNRAS, 443, 2264

Lombardi, M., Bouy, H., Alves, J., \& Lada, C. J. 2014, A\&A, 566, A45

Low, F. J., \& Aumann, H. H. 1970, ApJL, 162, L79

Marconi, A., Testi, L., Natta, A., \& Walmsley, C. M. 1998, A\&A, 330, 696

Martins, F., Schaerer, D., \& Hillier, D. J. 2005, A\&A, 436, 1049

Meixner, M., Haas, M. R., Tielens, A. G. G. M., Erickson, E. F., \& Werner, M. 1992, ApJ, 390, 499

Menten, K. M., Reid, M. J., Forbrich, J., \& Brunthaler, A. 2007, A\&A, 474, 515

Mizutani, M., Onaka, T., \& Shibai, H. 2004, A\&A, 423, 579

Muench, A. A., Lada, E. A., Lada, C. J., \& Alves, J. 2002, ApJ, 573, 366

Ney, E. P., Strecker, D. W., \& Gehrz, R. D. 1973, ApJ, 180, 809

O’Dell, C. R. 2001, ARA\&A, 39, 99

O’Dell, C. R., \& Bally, J. 1999, in ASP Conf. Ser. 188, Optical and Infrared Spectroscopy of Circumstellar Matter, ed. E. Guenther, B. Stecklum, \& S. Klose, 25

O’Dell, C. R., \& Doi, T. 2003, AJ, 125, 277

O'Dell, C. R., \& Harris, J. A. 2010, AJ, 140, 985

O’Dell, C. R., Henney, W. J., Abel, N. P., Ferland, G. J., \& Arthur, S. J. 2009, AJ, 137, 367
O’Dell, C. R., Muench, A., Smith, N., \& Zapata, L. 2008, Handbook of Star Forming Regions, Vol. I (San Francisco, CA: ASP)

O’Dell, C. R., \& Yusef-Zadeh, F. 2000, AJ, 120, 382

Ochsendorf, B. B., Cox, N. L. J., Krijt, S., et al. 2014a, A\&A, 563, A65

Ochsendorf, B. B., \& Tielens, A. G. G. M. 2015, A\&A, 576, A2

Ochsendorf, B. B., Verdolini, S., Cox, N. L. J., et al. 2014b, A\&A, 566, A75

Okada, Y., Pilleri, P., Berné, O., et al. 2013, A\&A, 553, A2

Ormel, C. W., Min, M., Tielens, A. G. G. M., Dominik, C., \& Paszun, D. 2011, A\&A, 532, A43

Ormel, C. W., Paszun, D., Dominik, C., \& Tielens, A. G. G. M. 2009, A\&A, 502,845

Ossenkopf, V., \& Henning, T. 1994, A\&A, 291, 943

Ossenkopf, V., Röllig, M., Neufeld, D. A., et al. 2013, A\&A, 550, A57

Pagani, L., Steinacker, J., Bacmann, A., Stutz, A., \& Henning, T. 2010, Sci, 329, 1622

Paladini, R., Umana, G., Veneziani, M., et al. 2012, ApJ, 760, 149

Parmar, P. S., Lacy, J. H., \& Achtermann, J. M. 1991, ApJL, 372, L25

Peeters, E., Martín-Hernández, N. L., Damour, F., et al. 2002, A\&A, 381, 571

Pellegrini, E. W., Baldwin, J. A., Ferland, G. J., Shaw, G., \& Heathcote, S. 2009, ApJ, 693, 285

Pilbratt, G. L., Riedinger, J. R., Passvogel, T., et al. 2010, A\&A, 518, L1

Planck Collaboration, Abergel, A., Ade, P. A. R., et al. 2011, A\&A, 536, A25

Planck Collaboration, Abergel, A., Ade, P. A. R., et al. 2014a, A\&A, 566, A55

Planck Collaboration, Abergel, A., Ade, P. A. R., et al. 2014b, A\&A, 571, A11

Poglitsch, A., Waelkens, C., Geis, N., et al. 2010, A\&A, 518, L2

Rathborne, J. M., Jackson, J. M., \& Simon, R. 2006, ApJ, 641, 389

Robberto, M., Beckwith, S. V. W., Panagia, N., et al. 2005, AJ, 129, 1534

Rubin, R. H., Simpson, J. P., O’Dell, C. R., et al. 2011, MNRAS, 410, 1320

Salgado, F., Berné, O., Adams, J. D., et al. 2012, ApJL, 749, L21

Sánchez, S. F., Cardiel, N., Verheijen, M. A. W., et al. 2007, A\&A, 465, 207

Shuping, R. Y., Morris, M., \& Bally, J. 2004, AJ, 128, 363

Shuping, R. Y., Morris, M. R., Herter, T. L., et al. 2012, ApJL, 749, L22

Simon, R., Stutzki, J., Sternberg, A., \& Winnewisser, G. 1997, A\&A, 327, L9

Simón-Díaz, S., Herrero, A., Esteban, C., \& Najarro, F. 2006, A\&A, 448, 351

Smith, C. H., Bourke, T. L., Wright, C. M., et al. 1999, MNRAS, 303, 367

Smith, N., Bally, J., Shuping, R. Y., Morris, M., \& Kassis, M. 2005, AJ, 130, 1763

Stahl, O., Wade, G., Petit, V., Stober, B., \& Schanne, L. 2008, A\&A, 487,323

Steinacker, J., Pagani, L., Bacmann, A., \& Guieu, S. 2010, A\&A, 511, A9

Stepnik, B., Abergel, A., Bernard, J.-P., et al. 2003, A\&A, 398, 551

Tielens, A. G. G. M. 2005, The Physics and Chemistry of the Interstellar Medium (Cambridge: Cambridge Univ. Press)

Tielens, A. G. G. M. 2008, ARA\&A, 46, 289

Tielens, A. G. G. M., \& Hollenbach, D. 1985, ApJ, 291, 722

Tielens, A. G. G. M., Meixner, M. M., van der Werf, P. P., et al. 1993, Sci, 262,86

van der Werf, P. P., Stutzki, J., Sternberg, A., \& Krabbe, A. 1996, A\&A, 313,633

Wada, K., Tanaka, H., Okuzumi, S., et al. 2013, A\&A, 559, A62

Wada, K., Tanaka, H., Suyama, T., Kimura, H., \& Yamamoto, T. 2009, ApJ, 702,1490

Weingartner, J. C., \& Draine, B. T. 2001, ApJ, 548, 296

Wen, Z., \& O'dell, C. R. 1995, ApJ, 438, 784

Werner, M. W., Gatley, I., Becklin, E. E., et al. 1976, ApJ, 204, 420

Wolfire, M. G., Hollenbach, D., McKee, C. F., Tielens, A. G. G. M., \& Bakes, E. L. O. 1995, ApJ, 443, 152

Wright, E. L. 1973, ApJ, 185, 569

Zubko, V., Dwek, E., \& Arendt, R. G. 2004, ApJS, 152, 211 